\title{
Stream insects and gastropods in the island of Gran Canaria (Spain)
}

\author{
A. N. Nilsson 1 \\ B. Malmqvist ${ }^{1}$ \\ M. Báez ${ }^{2}$ \\ J. H. Blackburn ${ }^{3}$ \\ P. D. Armitage ${ }^{3}$
}

Keywords : aquatic insects, gastropods, streams, Canary Islands.

The current status of the stream-living insects and gastropods of the island of Gran Canaria in the Atlantic Canary Islands is documented. Data from semiquantitative kick samples taken in 11 streams are supplemented by non-standardized collecting in the same and ten additional streams. The kick samples included some 9,000 specimens of 98 taxa, and in total 123 taxa were recorded from the studied streams in 1994 and 1995. The stream fauna was dominated by Diptera with some 80 taxa, followed by Coleoptera with 37 species known from the island. The kick samples yielded 11-36 species per stream and visit, whereas up to 48 species were scored when all collecting methods were included. Local diversity given as Fisher's $\alpha$ ranged from 3.2 to 10.3. Clustering showed only weak spatial patterns and resulted in much higher similarities among localities when based on the semiquantitative samples than when based on presence/absence including records from all collecting methods. Species distributions among localities were not significantly nested. The faunal similarity with the adjacent island of Tenerife is striking. Six of the Tenerife species are seemingly absent from Gran Canaria, whereas Gran Canaria has 13 species not found in Tenerife. Most of the 22 aquatic insect species in Gran Canaria only known from older records, prefer lentic habitats not included in our study. All except one of the five species endemic to Gran Canaria are considered extinct or on the edge of extinction. Increased future extinction rates are predicted as a response of the extreme habitat loss, with only three permanent streams known on the island today.

\section{Les insectes et les gastéropodes d'eau courante de l'île de Gran Canaria (Espagne)}

Mots-clés : insectes aquatiques, gastéropodes, cours d'eau, Iles Canaries.

La situation actuelle des populations d'insectes et de gastéropodes d'eau courante de l'île de Gran Canaria (Iles Canaries, Océan Atlantique) est évaluée. Des données obtenues par «kick samples» semi-quantitatifs et provenant de 11 cours d'eau ont été augmentées par des récoltes non standardisées, effectuées sur les mêmes cours d'eau et sur dix cours d'eau supplémentaires. Les «kick samples» ont collecté quelques 9.000 spécimens appartenant à 98 taxons et, au total, 123 taxons ont été recensés des cours d'eau étudiés en 1994 et 1995. La faune d'eau courante est dominée par les Diptères avec quelques 80 taxons, suivis des Coléoptères avec 37 espèces connues de l'île. Les «kick samples»» ont permis de recenser de 11 à 36 espèces par cours d'eau et date de prélèvement, tandis que jusqu'à 48 espèces sont dénombrées quand toutes les méthodes de prélèvement sont utilisées simultánément. La diversité spécifique locale selon $\alpha$ de Fisher varie de 3,2 à 10,3. Une classification hiérarchique révèle seulement de faibles structures spatiales et aboutit à des similarités entre stations beaucoup plus élevées lorsqu'elle est basée sur des prélèvements semi-quantitatifs plutôt que sur la présence/absence prenant en compte les citations obtenues par toutes les méthodes de prélèvement. Les liaisons espèces-stations ne sont pas significatives. La similarité faunistique avec l'île voisine de Ténérife est frappante. Six des espèces de Ténérife sont vraisemblablement absentes de Gran Canaria, tandis que cette dernière renferme 13 espèces non trouvées à Ténérife. La plupart des 22 espèces d'insectes aquatiques de Gran Canaria qui sont seulement connues par des citations anciennes, préfèrent des habitats lentiques non pris en compte dans notre étude. A l'exception de l'une d'entre elles, les cinq espèces endémiques de Gran Canaria sont considérées comme éteintes ou en voie d'extinction. Des taux d'extinction accrus sont à envisager à l'avenir, conséquence de l'extrême régression des habitats qui se traduit par le maintien, aujourd'hui, de seulement trois cours d'eau permanents connus.

\footnotetext{
1. Department of Animal Ecology, University of Umeå, SE-901 87 Umeă, Sweden.

2. Department of Zoology, University of La Laguna, 38206 La Laguna, Tenerife, Spain.

3. Institute of Freshwater Ecology, East Stoke, Wareham, Dorset BH20 6BB, England. 


\section{Introduction}

The Atlantic Canary Islands, off the coast of Moroc$\mathrm{co}$, harbour a relatively rich fauna of stream-living macroinvertebrates with high levels of local endemism in some taxa. This interesting fauna was first investigated more intensively through the expeditions made by the Finnish entomologists Richard Frey, Ragnar Stora and Håkan Lindberg (e.g. Frey 1936, Lindberg 1953 ; see Machado 1987a for a full bibliography). Besides a few later reports on selected orders or families (e.g. Báez 1985, Balke et al. 1990, Crosskey 1988, Machado $1987 \mathrm{~b}$ ), more recent documentation of the entire macroinvertebrate communities is so far limited to the island of Tenerife (Armitage et al. 1995, Malmqvist et al. 1993, 1995).

Of the other islands, the comparatively large and least isolated islands Fuerteventura and Lanzarote are relatively dry and are generally without permanent stream habitats (Marzol 1984). The westernmost islands La Gomera, Hierro and La Palma are wetter, but their faunas are poorly documented. Gran Canaria has a high natural potential for harbouring a fauna as rich as that of Tenerife, due to its relative large size, high precipitation, and low degree of isolation. However, chiefly due to water diversion and forestry, the number of Gran Canarian streams has dropped from 285 to 20 between 1933 and 1973 (Alfonso Pérez 1980, in Crosskey 1988). Today, the number of more permanent streams is even lower.

In order to document the current situation of the Gran Canarian streams and their fauna, three of us (Báez, Malmqvist \& Nilsson) visited the island on two occasions, first in March 1994 and then again in November 1995. Our major aim was to revisit stream localities known from the literature, as well as finding others that had not been documented earlier, and describe their current macroinvertebrate fauna.

\section{Material and methods}

Gran Canaria was visited for collecting during two periods : 27 March to 1 April 1994 and 16-21 November 1995. Invertebrates were collected using a combination of different techniques : hand-nets, kick-sampling, direct picking, and turning of stones. Care was taken to include all possible microhabitats. Besides this qualitative sampling, semiquantitative net samples were taken in the larger streams. The sampling procedure involved taking 30s kick samples over one $\mathrm{m}^{2}$ areas with a small handnet. When possible three samples were taken in riffles and three in stream pools at each locality. Samples were taken so that the number of microhabitats was maximized and always included parts of the margins.

Small portable light traps were used on the following occasions in order to get adults of Trichoptera and Diptera to support larval identifications : Azuaje 31.iii.1.iv.1994 20.00-09.30 and 17-18.xi.1995 18.10-08.40; Mina 2 19-20.xi.1995 17.00-09.30; Soria $128-$ 29.iii.1994 18.00-09.30; Tirajana 30.iii.1994 20.0024.00, 2.i.1995 18.30-21.30, and 16.xi.1995 18.3021.30 .

Discharge was estimated as the product of mean depth, width, and surface velocity corrected with a coefficient of 0.67 to give mean velocity. Conductivity, $\mathrm{pH}$ and water temperature were measured directly in the field. Macroinvertebrate samples were sorted immediately or preserved individually in $70 \%$ ethanol and sorted out from debris later in the same day.

We used Fisher $\alpha$ as a measure of diversity that is independent of sample size (Magurran 1988, Rosenzweig 1995). This diversity index is based on the assumption that the abundances of species fit a log-series distribution. Small, incomplete samples of other distributions almost fit the log-series.

Similarity among stream localities was analysed by tree clustering. The purpose of this algorithm is to join objects into successively larger clusters, using some measure of similarity or distance. We used the Unweighted pair-group average (UPGMA) algorithm, in which the distance between two clusters is calculated as the average distance between all pairs of objects in the two different clusters.

Nestedness analysis (Patterson \& Atmar 1986) was used to test for structure in the distribution of species among streams. If distributions were strictly nested, each species would occur in all streams that have fewer species than the richest one in which it occurred. Deviations from this pattern were scored by summing all absences from poorer streams over all species. The significance of the resulting index was then tested against randomized data (RANDOM 1).

\section{Studied localities}

The studied localities (Fig. 1) are listed in Tab. 1 and selected chemical and physical characteristics are given in Tab. 2. All localities sampled in 1994 were revisited in 1995 and sampled again if carrying water. The following sites were totally dry in 1995 : Brezal, Hondo de Abajo, Hornos, Mina 1 \& 3, Peréz dam, Soria 1 $\& 2$. At the following sites no running water was found in 1995, but isolated pools were sampled qualitatively: Coruña, Moya, Soria 3, and Tirajana. 


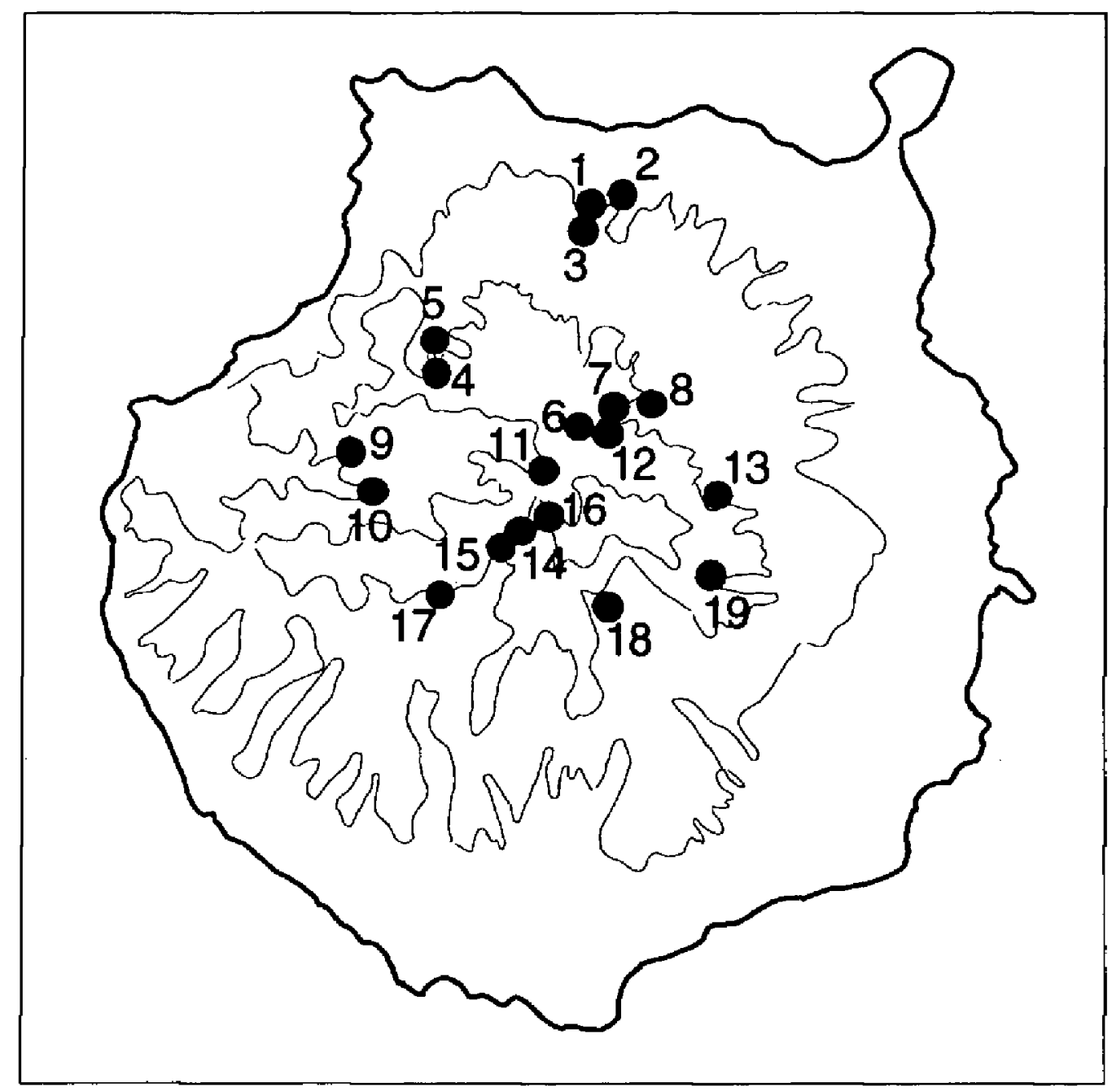

Fig. 1. Map of Gran Canaria with the position of our study sites, coded as : (1) Moya, (2) Azuaje, (3) Brezal, (4) Coruña, (5) Pérez dam, (6) Mina 1, (7) Mina 2, (8) Mina 3, (9) Carrizal 1-2, (10) Siberio, (11) Tejeda, (12) Gañanías, (13) Cernicalos, (14) Soria 1, (15) Soria 2, (16) Los Hornos, (17) Soria 3, (18) Tirajana, (19) Guayadeque. Fine lines show altitudes of 500,1000 and $1500 \mathrm{~m}$ asl.

Fig. 1. Carte de Gran Canaria avec emplacement des 19 sites étudiés. Les lignes fines figurent les altitudes 500,1000 et $1500 \mathrm{~m}$.

\section{List of limnic gastropods and insects known from Gran Canaria}

Distributions are given directly after the species names, and coded as : (GC) endemic to Gran Canaria, (Ca) endemic to the Canary Islands ( $F=$ Fuerteventura, $\mathrm{G}=$ Gomera, $\mathrm{H}=$ Hierro, $\mathrm{L}=$ Lanzarote, $\mathrm{LP}=\mathrm{La}$ Palma, $\mathrm{T}=$ Tenerife), (Ma) endemic to Macaronesia (Madeira plus the Canary Islands), (Me) Mediterranean, (WP) West Palearctic, (Af) Africa, (Am) America, and (Co) cosmopolitan.

The following list includes all species of the treated groups that have been recorded from Gran Canaria. Our species records from the semiquantitative sampling localities are given in Tab. 3. Presence of species at the rest of the localities is given in Tab. 4.

\subsection{Gastropoda}

Ancylidae

Ancylus striatus Quoy \& Gaimard, $1833 \mathrm{Ma}$

This species was found in all but two of the streams in which semiquantitative net samples were taken (Tab. 3). Also found at all but five of the qualitative sample sites (Table 4). The sites where it was absent tended to carry very little water or had no flow at all.

Physidae

Physella acuta (Draparnaud, 1805) Me (Co)

This was the most widespread gastropod species in Gran Canaria, and we found it in all streams in which semiquantitative net samples were taken, except Soria 1 (Table 3). Also found in qualitative samples at : Carrizal 1-2, Hondo de Abajo, and Soria 3. The sites where it was absent tended to carry very little water or had no flow at all. 
Table 1. List of the studied stream localities in Gran Canaria with their altitudes in $\mathrm{m}$ asl and a general description of the sampled habitats

Tableau 1. Liste des sites étudiés de Gran Canaria avec leurs altitudes en $m$ et une description générale des habitats.

\begin{tabular}{llcll}
\hline Barranco & Locality & Altitude & Habitat & Date \\
\hline Azuaje & $1 \mathrm{~km}$ W Firgas & 240 & stream & $940401 \& 951117$ \\
Brezal & $2 \mathrm{~km}$ ESE Moya, nr Los Tilos & 570 & canal & 940331 \\
Carrizal 1 & $7 \mathrm{~km}$ ENE San Nicolás, nr Parrallilo dam & 290 & small stream & 951120 \\
Carrizal 2 & $6 \mathrm{~km}$ ENE San Nicolás & 250 & small stream & 951120 \\
Cernicalos & $6 \mathrm{~km}$ WSW Telde at Lomo Magullo & 570 & stream & $940327 \& 951116$ \\
Coruña & $3 \mathrm{~km}$ NW Artenara & 980 & stream & $940330 \& 951118$ \\
Gañanías & $\mathrm{nr}$ Las Lagunetas & 1250 & wet rock & $940328 \& 951119$ \\
Guayadeque & $7 \mathrm{~km}$ WNW Ingenio & 970 & trickle & $940327 \& 951116$ \\
Hondo de Abajo & $6.5 \mathrm{~km}$ SE Agaete, nr Pérez dam & 825 & small stream & 940330 \\
Hornos & Los Hornos & 1640 & trickle & 940329 \\
Mina 1 & Hoya de la Montana & 1390 & cascade & 940328 \\
Mina 2 & $2 \mathrm{~km}$ SW Las Lagunetas & 1350 & stream & 951119 \\
Mina 3 & $\mathrm{nr}$ Utiaca & 770 & trickle & 940328 \\
Moya & $\mathrm{nr}$ Moya & 290 & trickle & $940331 \& 951117$ \\
Pérez dam & $6.5 \mathrm{~km}$ SE Agaete & 820 & wet rock & 940330 \\
Siberio & $9 \mathrm{~km}$ ESE San Nicolás & 450 & trickle & 951120 \\
Soria 1 & $1 \mathrm{~km}$ W Ayacata & 1370 & trickle & $940329 \& 951118$ \\
Soria 2 & $4 \mathrm{~km}$ SW Ayacata & 925 & stream & 940329 \\
Soria 3 & $6 \mathrm{~km}$ SW Ayacata, nr Niñas dam & 910 & stream & $940329 \& 951118$ \\
Tejeda & $1 \mathrm{~km}$ SSE Tejeda & 1040 & stream & $940328 \& 951118$ \\
Tirajana & $\mathrm{nr}$ San Bartolomé de Tirajana & 890 & stream & $940329 \& 951116$ \\
\hline
\end{tabular}

Lymnaeidae

Galba cf. cubensis (Pfeiffer, 1839) Am

Besides in five of the streams in which semiquantitative net samples were taken (Table 3), this species was also found at Guayadeque.

Pseudosuccinea columella (Say, 1817) Am

Besides in four of the streams in which semiquantitative net samples were taken (Table 3), this species was also found at Carrizal 1 and 2.

Planorbiidae

Gyraulus parvus (Say, 1817) Am

This species was collected in six of the streams mentioned in Table 3. At Azuaje, only a few specimens were hand-collected.
Planorbella duryi (Wetherby, 1879) Am

This species was only found at Azuaje, where it seemingly was more abundant in 1995 than in 1994 (Table 3).

Hydrobiidae

The unidentified species we have found most probably belongs to the genus Hydriobia (Proschwitz, in litt). All our specimens were found at Azuaje in 1995.

\subsection{Ephemeroptera}

Baetidae

Baetis canariensis Müller-Liebenau, 1971 Ca (G LP T)

This Canarian endemic was first described from Bco. de la Mina at $1,150 \mathrm{~m}$ asl, and Müller-Liebenau (1971) also reported it from Bco. Los Propios at $500 \mathrm{~m}$ and Bco. Agaete at $450-700 \mathrm{~m}$. We found it abundant at Cernicalos and Mina 2, 
Table 2. Selected chemical and physical characteristics of the water in the streams. Variables coded as: (Cond.) conductivity in ms/cm, (Temp.) temperature in ${ }^{\circ} \mathrm{C}$, (Flow) flow of stream in $\mathrm{L} / \mathrm{s}$.

Tableau 2. Caractéristiques physiques et chimiques de l'eau dans les cours d'eau le jour de notre visite. Codes des variables : (Cond.) conductivité en ms/cm, (Temp.) température en ${ }^{\circ} \mathrm{C}$, (Flow.) débit en $\mathrm{L} / \mathrm{s}$.

\begin{tabular}{|c|c|c|c|c|c|}
\hline Locality & Date & Cond. & $\mathrm{pH}$ & Temp. & Flow \\
\hline \multirow[t]{2}{*}{ Azuaje } & 940401 & 2090 & 7.45 & 15.4 & 2 \\
\hline & 951117 & 2820 & 8.1 & 18.3 & 13 \\
\hline Brezal & 940331 & 971 & 8.82 & 17.8 & 0.5 \\
\hline Carrizal 1 & 951120 & 744 & 7.88 & 19.4 & 0.1 \\
\hline Carrizal 2 & 951120 & & & & 5 \\
\hline \multirow[t]{2}{*}{ Cernicalos } & 940327 & 233 & 7.65 & 13.3 & 13 \\
\hline & 951116 & 241 & 7.85 & 16.7 & 12 \\
\hline \multirow[t]{2}{*}{ Coruña } & 940330 & 608 & 7.65 & 12.5 & 17 \\
\hline & 951118 & 801 & 6.54 & 16.5 & 0 \\
\hline \multirow[t]{2}{*}{ Gañanias } & 940328 & 136 & 7.23 & 13.3 & 10 \\
\hline & 951119 & 181 & 7.9 & 12.8 & $<1$ \\
\hline \multirow[t]{2}{*}{ Guayadeque } & 940327 & 210 & 7.61 & 16.4 & 0.05 \\
\hline & 951116 & 205 & 7.84 & 18 & 0.04 \\
\hline Hondo de Abajo & 940330 & 614 & 7.57 & 15.9 & 1 \\
\hline Hornos & 940329 & 279 & 7.44 & 9.2 & 0.1 \\
\hline Mina 1 & 940328 & 385 & 8.39 & 15.3 & 15 \\
\hline Mina 2 & 951119 & 136 & 7.96 & 11.6 & 18 \\
\hline Mina 3 & 940328 & 913 & 7.01 & 14.9 & 0.3 \\
\hline \multirow[t]{2}{*}{ Moya } & 940331 & 1130 & 7.79 & 16 & 1 \\
\hline & 951117 & 1569 & 8.35 & 25.4 & 0 \\
\hline Pérez dam & 940330 & 511 & 7.99 & 13.4 & 1 \\
\hline Siberio & 951120 & 603 & 7.4 & 18.1 & 0 \\
\hline Soria 1 & 940329 & 249 & 7.92 & 11.8 & 0.1 \\
\hline Soria 2 & 940329 & 330 & 8.14 & 18.2 & 10 \\
\hline \multirow[t]{2}{*}{ Soria 3} & 940329 & 314 & 8.61 & 16.8 & 2 \\
\hline & 951118 & 1109 & 8.29 & 15 & 0 \\
\hline \multirow[t]{2}{*}{ Tejeda } & 940328 & 604 & 8.3 & 18.9 & 35 \\
\hline & 951118 & 1296 & 7.26 & 19.5 & $<1$ \\
\hline Tirajana & 940329 & 243 & 7.05 & 15.7 & 3 \\
\hline
\end{tabular}

and present also at Soria $2-3$, Tejeda and Tirajana (Tables 3-4).

Baetis nigrescens Navas, $1932 \mathrm{Me}$

This species was first described from Los Tilos in Gran Canaria, and later reported from Bco. de la Mina at $1,150 \mathrm{~m}$ and Bco. Los Propios at 500m (Müller-Liebenau 1971). We found it in the semiquantitative samples at the following localities (Table 3) : Azuaje, Cernicalos, Mina 2, Tejeda and Tirajana. Baetis pseudorhodani Müller-Liebenau, 1971 Ca (G LP T)

Müller-Liebenau (1971) described this species from the same site as $B$. canariensis, and found it also in Bco. Agaete at Los Berrazales. We found it abundantly at Cernicalos and Mina 2, and present also at Mina 3, Soria 3, and Tejeda (Tables 3-4).

\section{Cloeon dipterum (Linnaeus, 1761) WP}

Besides the six localities given in Tab. 3, this species was also found at Carrizal 1 \& 2, Hondo de Abajo, and Soria 3 (Table 4).
Cloeon sp. 1 (sensu Malmqvist et al: 1995)

Specimens assigned to this species were found in qualitative samples from Cernicalos and Coruña (Tables 3-4).

Cloeon sp. 2 (sensu Malmqvist et al. 1995)

We collected this species at the following stream sites : Azuaje, Carrizal 1, Coruña, Hondo de Abajo, Hornos, Moya, Siberio, Soria 2-3, and Tirajana (Tables 3-4).

Caenidae

Caenis luctuosa (Burmeister, 1839) WP

This species was abundant at Azuaje, and occasional larvae were found also at Carrizal 1 \& 2 and Siberio (Tabs 3-4).

\subsection{Odonata}

Coenagrionidae

Ischnura saharensis Aguesse, 1968 Af

This species was first recorded from Gran Canaria by Valle (1955; Aldea San Nicolás) misidentified as I. senegalensis. 
Table 3. Contents of net samples from selected Gran Canaria streams in 1994 and 1995 . Values represent three pooled samples from riffles (R) or pools (P). Presence in qualitative samples given with « $\mathrm{X} »$ is not included in the summations.

Tableau 3. Contenus des récoltes au filet des cours d'eau de Gran Canaria visités en 1994 et 1995 . Les chiffres représentent trois récoltes de radiers $(R)$ et de mouilles (P). La présence dans des récoltes qualitatives marquée par «X» n'est pas comptabilisée dans les totaux.

\begin{tabular}{|c|c|c|c|c|c|c|c|c|c|c|c|c|c|c|c|c|}
\hline \multirow[t]{3}{*}{ Taxa } & \multicolumn{4}{|c|}{ Azuajo } & \multicolumn{4}{|c|}{ Cemicalos } & \multirow{2}{*}{\multicolumn{2}{|c|}{$\begin{array}{l}\text { Coruña } \\
94\end{array}$}} & \multirow{2}{*}{\multicolumn{2}{|c|}{$\begin{array}{l}\text { Mina } 2 \\
95\end{array}$}} & \multirow{2}{*}{\multicolumn{2}{|c|}{$\begin{array}{l}\text { Mina 3 } \\
\cdot 94\end{array}$}} & \multirow{2}{*}{\multicolumn{2}{|c|}{ Moya }} \\
\hline & 94 & & 95 & & 94 & & 95 & & & & & & & & & \\
\hline & $\mathbf{R}$ & $\mathbf{P}$ & $\mathbf{R}$ & $\mathbf{P}$ & $\mathbf{A}$ & $\mathbf{p}$ & $\mathbf{R}$ & $\mathbf{P}$ & $\mathrm{R}$ & $\mathbf{P}$ & A & $\mathbf{p}$ & $\mathbf{A}$ & $\mathbf{P}$ & $\mathbf{A}$ & $\mathbf{P}$ \\
\hline \multicolumn{17}{|l|}{ Gestropoda } \\
\hline Ancylus striatus & 29 & 3 & 5 & 6 & 4 & 6 & 8 & 1 & 2 & 1 & 8 & 1 & & & 114 & 5 \\
\hline Physella acuta & 24 & 74 & 18 & 20 & & 14 & 14 & 68 & & & & 1 & 1 & 37 & 7 & 13 \\
\hline Galba cf. cubensis & & 2 & & & & & & & & 2 & & & & & 1 & 4 \\
\hline Pseudosuccinea columella & 1 & & 3 & & & & 2 & 5 & & & & & & & & \\
\hline Gyraulus parus & & $x$ & & & 2 & 11 & 3 & 42 & & 1 & & & & & & 1 \\
\hline Planorbella duryl & 3 & 4 & 58 & 62 & & & & & & & & & & & & \\
\hline Hydrobiidae indet & & & 7 & & & & & & & & & & & & & \\
\hline \multicolumn{17}{|l|}{ Ephemeroptera } \\
\hline Baetis cananiensis & & & & & 299 & 300 & 70 & 40 & & & 43 & 15 & & & & \\
\hline Baetis nigrescens & 3 & & 4 & & 3 & & 14 & & & & 1 & & & & & \\
\hline Baetis pseudorhodanI & & & & & 53 & $\mathbf{9}$ & 23 & 3 & & & 33 & & & $x$ & & \\
\hline Cloeon dipterum & & $x$ & & 22 & & & & & & 5 & & & & & & 20 \\
\hline Cloeon sp. 1 & & & & & & $\mathbf{x}$ & & & & & & & & & & \\
\hline Cloeon sp. 2 & & $x$ & & 1 & & & & & & 9 & & & & & & 12 \\
\hline Caenis luctuasa & 1 & 1 & 29 & 9 & & & & & & & & & & & & \\
\hline \multicolumn{17}{|l|}{ Odonata } \\
\hline Libellulidae indet. & & 4 & & & & & & & & & & & & & & 18 \\
\hline Ortherrum chrysostigma & & & 2 & & & & & & & & & & & & & \\
\hline Sympetrum lonscolombei & & $x$ & & & & & & & & & & & & & & \\
\hline Trithemis arteriasa & & & & & & & & & & & & & & & & 2 \\
\hline Zygonyx torrida & & & 2 & & & & & & & & & & & & & \\
\hline \multicolumn{17}{|l|}{ Heteroptera } \\
\hline Corixa affinis & & & & & & & & & & $x$ & & & & & & 2 \\
\hline Notonecta canariensis & & $x$ & & & & & & & & & & & & $x$ & & 4 \\
\hline Hydrometra stagnorum & & & & & & $x$ & 1 & 3 & & & & & & & & \\
\hline Microvelia gracillima & & $x$ & 2 & 1 & & & & & & & & & & & & $x$ \\
\hline Vella lindbergi & & & & & 12 & 4 & & & & & & & & 1 & & \\
\hline \multicolumn{17}{|l|}{ Coleoptera } \\
\hline Gyrinus dejeani & & $x$ & & & & & & 18 & & $x$ & & & & & & $x$ \\
\hline Grinus urinator & & & & & & & & 1 & & & & & & & & \\
\hline Haliplus fineatocolfis & 5 & 5 & & 6 & & & & & & 3 & & & & & 1 & 3 \\
\hline Bidessus minutissimus & & $x$ & 1 & 1 & & & & & & & & & & & & \\
\hline Herophydrus musicus & & $\mathbf{x}$ & & & & & & & & & & & & & & 6 \\
\hline Hyphydrus maculatus & & & & & & & & & & & & & & & & 2 \\
\hline Hydrotarsus pilosus & & & & & & & & & & & & & & & 1 & \\
\hline Hydroporus errans & & 1 & & & & 6 & & & 5 & & & & & 9 & 1 & 4 \\
\hline Graptodytes delectus & & & & & & & & & & & & & & & & $x$ \\
\hline Stictonectes canariensis & & & & & & & & & & 9 & & & & & 1 & \\
\hline Nebriopons canariensis & & & & & & 7 & & & & & & & & 1 & & \\
\hline Agabus biguttatus & & & & & & $x$ & & & 6 & 16 & & & 26 & 20 & 1 & 1 \\
\hline Agabus nabulosus & & 2 & & & & 1 & & & & & & & & $x$ & & 4 \\
\hline Meladema coriacea & & & & & & & & & & 8 & & & & & & 1 \\
\hline Laccophilus hyalinus & & 15 & & 3 & & & & & & & & & & & & 11 \\
\hline Ochthabius rugulosus & & & & $x$ & & $x$ & & & & & & & & & & $x$ \\
\hline Limnebius graclipes & & & & & & $x$ & & & & $x$ & & & & & & \\
\hline Mydrochus grandicollis & & & & & & & & & & & & & & 1 & & \\
\hline Coelostoma hispanioum & & $x$ & & & & & & & & & & & & & & \\
\hline Laccobius canariensis & & & & & & & & & & $x$ & & & & & & $x$ \\
\hline Anacaena haemorrhoa & & $x$ & & & 1 & 1 & 2 & & & 1 & & & & & & \\
\hline Helochares lividus & & $x$ & & $x$ & & & & & & & & & & & & 2 \\
\hline Dryops gracilis & & & 5 & & 19 & 22 & 24 & 18 & 1 & 3 & & 1 & & 1 & 1 & 1 \\
\hline Trichoptera & & & & & & & & & & & & & & & & \\
\hline Agapetus adejensis & & & & & 7 & & 6 & & & & & & & & & \\
\hline
\end{tabular}


Table 3 (continued). Contents of net samples from selected Gran Canaria streams in 1994 and 1995 . Values represent three pooled samples from riffles $(\mathrm{R})$ or pools $(\mathrm{P})$. Presence in qualitative samples given with $« \mathrm{x} \otimes$ is not included in the summations.

Tableau 3 (suite). Contenus des récoltes au filet des cours d'eau de Gran Canaria visités en 1994 et 1995 . Les chiffres représentent trois récoltes de radiers $(R)$ et de mouilles $(P)$. La présence dans des récoltes qualitatives marquée par « $x » n$ 'est pas comptabilisée dans les totaux.

\begin{tabular}{|c|c|c|c|c|c|c|c|c|c|c|c|c|c|c|c|c|}
\hline \multirow[t]{3}{*}{ Taxa } & \multicolumn{4}{|c|}{ Azuaje } & \multicolumn{4}{|c|}{ Cemicalos } & \multirow{2}{*}{\multicolumn{2}{|c|}{$\begin{array}{l}\text { Coruña } \\
94\end{array}$}} & \multirow{2}{*}{\multicolumn{2}{|c|}{$\begin{array}{l}\text { Mina } 2 \\
95\end{array}$}} & \multirow{2}{*}{\multicolumn{2}{|c|}{$\begin{array}{l}\text { Mina } 3 \\
94\end{array}$}} & \multirow{2}{*}{\multicolumn{2}{|c|}{$\begin{array}{l}\text { Moya } \\
94\end{array}$}} \\
\hline & 94 & & 95 & & 94 & & 95 & & & & & & & & & \\
\hline & $\mathbf{R}$ & $\mathbf{P}$ & $\mathbf{R}$ & $\mathbf{P}$ & A & $\mathbf{P}$ & $\mathbf{A}$ & $\mathbf{P}$ & A & $\mathbf{P}$ & $\mathbf{R}$ & $\mathbf{P}$ & $\mathbf{R}$ & $\mathbf{P}$ & $\mathbf{A}$ & $\mathbf{P}$ \\
\hline Hydroptila fortunata & 38 & 33 & 17 & 8 & 9 & 7 & 2 & 1 & & & & & & & 7 & 7 \\
\hline Orthotrichia angustella & & & 1 & 2 & & & & & & & & & & & & \\
\hline Oxyethira sp. & & 3 & & & & & & & & & & & & & & $x$ \\
\hline Hydropsyche maroccana & 1 & & & & 29 & & 10 & 1 & & & 28 & 6 & & $x$ & & \\
\hline Mesophylax aspersus & & & & & 19 & 52 & 9 & 39 & 19 & 46 & 2 & 29 & $\pi$ & 8 & & \\
\hline \multicolumn{17}{|l|}{ Diptera } \\
\hline Limoniidae indet & & & & & & & & & & & & & 16 & 1 & & $x$ \\
\hline Psychodidae indet & & $x$ & 1 & & & & & 1 & & 2 & & & 6 & & & \\
\hline Dixa tetrica & & & & & & 9 & 2 & 6 & 3 & 1 & & 1 & & 4 & 4 & 1 \\
\hline Anopheles sp. & & & & & & & & & & & & & & & & $x$ \\
\hline Culiseta longiareolata & & & & & & $x$ & & & & & & & & 7 & & \\
\hline Culex sp. & & & & & & $x$ & & & & $x$ & & & & & & \\
\hline Thaumalea subatricana & & & & & & & & & & & & $x$ & & & & \\
\hline Simulium guimari & & & & & 91 & & 89 & 14 & & & 39 & & 3 & & & $x$ \\
\hline Simulium intermedium & 153 & & & & & & & & 810 & & 127 & 1 & 2 & 1 & 60 & 2 \\
\hline Simulium pseudequinum & 167 & & & & & & & & & & & & & & & \\
\hline Simulium ruficome & 141 & & 188 & & & & & & & & & & & & 53 & 2 \\
\hline Ceratopogonidae indet. & & $x$ & & & & & & & & & & & & & & \\
\hline Ablabesmyia dusoleili & & 10 & 3 & & 4 & 18 & 1 & & & & & & & & 1 & 1 \\
\hline Macropelopia nebulosa & & & & & & & & & & 1 & & & & & & \\
\hline Macropelopia sp. & & & & & & & & & & 2 & & & 1 & 5 & & \\
\hline Paramerina cingulata & & 2 & & 1 & & 1 & & & & & & & & 2 & & \\
\hline Procladius choreus & & & & & & & & & & & & & & 1 & & 1 \\
\hline Thienemannimyia sp. & & & & & & & 1 & & & & & & & & & \\
\hline Zavrelimyia babatipes & & 1 & & & & 1 & & & & 21 & & & 1 & 89 & 2 & 2 \\
\hline Cardjocladius sp. & & & & & & & 2 & & & & & $x$ & & & & \\
\hline Chaetocladius sp. & & & & & & & & & & & & & 37 & & & \\
\hline Connoneura sp. & & & & & & & & & 1 & & & 1 & 8 & & & \\
\hline Eukiefferiella minoor & & & & & & & & & 2 & & & & 135 & & & \\
\hline Eukiefferialla sp. nr ilkJeyensis & 4 & & & & & & & & & & 1 & & & & & \\
\hline Eukjefferella indet & & & & & & & & & & & & & 2 & & & \\
\hline Limnophyes sp. & & & & & & & & & & & & & 10 & & 1 & \\
\hline Orthocladius (Eudactylocladius) fuscimanus & & & & & & & & & & & & & 299 & & & \\
\hline Orthocladius (Euorthocladius) ivicola & & & & & & & & & 57 & & & & 34 & & & \\
\hline Orthocladlus (Orthocladius) pedestris & & & & & & & & & 5 & 79 & & $\mathbf{x}$ & & 13 & & \\
\hline Parametriocnemus stylatus & 3 & & 42 & & & & 1 & & & 1 & 3 & & & & 2 & \\
\hline Paratrichocladius sp. & 40 & 4 & & & 1 & & & & 12 & 207 & & & 26 & 32 & 5 & \\
\hline Aheocricotopus atripes & 1 & & & & & & & & 5 & 1 & & & 15 & 1 & & \\
\hline Thienemanniella sp. nr clavicornis & & & & & 2 & & 1 & & 3 & 1 & 5 & 4 & 7 & & & \\
\hline Chironomus sp. & & $x$ & & & & $x$ & & & & & & & & 102 & & 1 \\
\hline Phaenopsectra sp. & & 1 & & & & & & & & & & & & 7 & & \\
\hline Polypedilum sp. & & & & & & & & & & & & & & & & 1 \\
\hline Cladotanytarsus sp. & & & & & & & & & & & & & & 1 & & 3 \\
\hline Micropsectra sp. & & & & & & & & & 1 & 11 & & & 226 & 18 & & \\
\hline Rheotanytarsus sp. & 1 & & 13 & & 1 & & 1 & & & & & & & & & \\
\hline Tanytarsus sp. & & & & & & & & & & & & & & & 1 & 3 \\
\hline Virgatanytarsus sp. & & & 1 & & & & & & & & & & & & & \\
\hline Tanytarsini indet. & & & & & & & & & & & & & 2 & & & \\
\hline Oxycera grancanariensis & & 1 & & & & $x$ & & 2 & & & & & & 1 & & \\
\hline Empididae indet & & & & & & & & & & 11 & & & 6 & 3 & & \\
\hline Limnophora sp. & & $x$ & & & & & & & & & & & & & & $x$ \\
\hline No. of Inds. & 615 & 166 & 402 & 142 & 556 & 469 & 286 & 263 & 932 & 442 & 290 & 60 & 940 & 366 & 264 & 140 \\
\hline No. of taxa & 17 & 18 & 20 & 13 & 17 & 17 & 22 & 17 & 15 & 24 & 11 & 10 & 22 & 25 & 19 & 31 \\
\hline
\end{tabular}


Table 3 (continued). Contents of net samples from selected Gran Canaria streams in 1994 and 1995. Values represent three pooled samples from riffles (R) or pools (P). Presence in qualitative samples given with $\langle x »$ is not included in the summations.

Tableau 3 (suite). Contenus des récoltes au filet des cours d'eau de Gran Canaria visités en 1994 et 1995. Les chiffres représentent trois récoltes de radiers $(\mathrm{R})$ et de mouilles $(\mathbf{P})$. La présence dans des récoltes qualitatives marquée par « $\mathrm{n} »$ n'est pas comptabilisée dans les totaux.

\begin{tabular}{|c|c|c|c|c|c|c|c|c|c|c|c|}
\hline \multirow[t]{3}{*}{ Taxa } & \multirow{3}{*}{$\begin{array}{l}\text { Siberio } \\
95 \\
P \\
\end{array}$} & \multicolumn{2}{|c|}{ Soria 1} & \multicolumn{2}{|c|}{ Soria 2} & \multicolumn{2}{|c|}{ Tejeda } & \multirow[b]{2}{*}{95} & & \multicolumn{2}{|c|}{ Tirajana } \\
\hline & & 94 & & 94 & & 94 & & & & 94 & \\
\hline & & $\mathbf{R}$ & $\mathbf{P}$ & $\mathbf{R}$ & $P$ & $\mathbf{A}$ & $\mathbf{P}$ & $\mathbf{R}$ & $\mathbf{P}$ & $\mathbf{R}$ & $\mathbf{P}$ \\
\hline \multicolumn{12}{|l|}{ Gestropoda } \\
\hline Ancylus strlatus & $x$ & & & 3 & 19 & & 3 & 14 & 1 & 23 & 23 \\
\hline Physella acuta & 65 & & & & 3 & & 1 & 82 & 74 & 4 & 18 \\
\hline Galba cf. cubensis & & & & 1 & & & 2 & & 1 & & \\
\hline Pseudosuccinea columella & 13 & & & & & & & & & & 1 \\
\hline Gyraulus pamus & 13 & & & & & & & 5 & & & \\
\hline \multicolumn{12}{|l|}{ Ephemeroptera } \\
\hline Bagtis canariensis & & & & 1 & & 1 & & & & 1 & \\
\hline Battis nigrescens & & & & & & 1 & & & & 6 & \\
\hline Baetis pseudorhodant & & & & & & 1 & & 1 & & & \\
\hline Clooon dipterum & 17 & & 1 & & & & 3 & 1 & 15 & & \\
\hline Cboon sp. 2 & $x$ & & & & $x$ & & & & & & 1 \\
\hline Caenis Iuctuosa & 2 & & & & & & & & & & \\
\hline \multicolumn{12}{|l|}{ Odonata } \\
\hline Ischnura saharensis & $x$ & & & & & & & & & & \\
\hline Libellulidae indet. & 7 & & & & $x$ & & & & 1 & & 1 \\
\hline Orthetrum chnysostlgma & 5 & & & & & & & 18 & 1 & & \\
\hline Crocothemis enythraea & 19 & & & & & & & & 5 & & \\
\hline Trithemis arteriosa & 1 & & & & & & & & & & \\
\hline \multicolumn{12}{|l|}{ Heteroptera } \\
\hline Corixa affinis & $x$ & & & & & & & & & & \\
\hline Sigara bteralis & $x$ & & & & & & & & & & \\
\hline Notonecta canariensis & $x$ & & & & $x$ & & 3 & & & & 1 \\
\hline Hebrus pusillus & $\mathbf{x}$ & & & & & & $\mathrm{x}$ & & & & \\
\hline Microvelia gracillima & 2 & & & & & & & 1 & 1 & & \\
\hline \multicolumn{12}{|l|}{ Coleoptera } \\
\hline Gyrinus dejeani & $x$ & & & & 1 & & $x$ & & & & $x$ \\
\hline Gyrinus urinator & 4 & & & & & & & & & & \\
\hline Haliplus lineatocolis & 10 & & & & 2 & & 2 & 3 & 3 & & 6 \\
\hline Bidessus minutissimus & $x$ & & & & $x$ & & 2 & & & & 20 \\
\hline Hydroporus errans & & 1 & 3 & & $x$ & & 1 & 1 & 6 & & $x$ \\
\hline Graptodytes delectus & $x$ & & & & $\mathrm{x}$ & & $x$ & & & & \\
\hline Stictonectes canariensis & $x$ & & & & 11 & & $x$ & & & & 3 \\
\hline Agabus biguttatus & $x$ & & & & 1 & & $x$ & 1 & & & \\
\hline Meladema coriacea & $x$ & & & & $x$ & & 1 & & & & \\
\hline Laccophilus hyalinus & 1 & & & & & & 3 & & 13 & & \\
\hline Ochthebius quadrifoveolatus & & & & & $x$ & & 1 & & 1 & 1 & 1 \\
\hline Ochthebius rugulasus & & & $x$ & & & & & & & & $x$ \\
\hline Hydraena serricollis & $x$ & & & & & & & & & & $x$ \\
\hline Limnebius canariensis & & & & & $x$ & & & & & & \\
\hline Limnebius gracilipes & $x$ & & & & $x$ & & $x$ & & & & $x$ \\
\hline Hydrochus grandicollis & $x$ & & & & $x$ & & 1 & & & & \\
\hline Coolostoma hispanicum & $x$ & & & & & & $x$ & & & & \\
\hline Chaetarthria similis & & & & & & & $x$ & & & & \\
\hline Laccobius canariensis & 2 & & & & & & $x$ & & 5 & & $x$ \\
\hline Laccobius gracilis & & & & & & & $\mathrm{x}$ & & & & \\
\hline Helochares lividus & $x$ & & & & $x$ & & $x$ & & & & \\
\hline Dryops gracilis & $\mathbf{x}$ & & 1 & & $x$ & 2 & 2 & 6 & 14 & 1 & 2 \\
\hline \multicolumn{12}{|l|}{ Trichoptere } \\
\hline Hydroptila fortunata & $x$ & & & & 1 & 16 & 4 & 56 & & 31 & 32 \\
\hline Oxyethira sp. & 1 & & & & & & & & & & \\
\hline Polycentropus sp. & $x$ & & & & & & & & & & 1 \\
\hline Mesophylax aspersus & & 33 & 1 & 2 & 3 & 15 & 17 & & & 2 & 6 \\
\hline
\end{tabular}


Table 3 (continued). Contents of net samples from selected Gran Canaria streams in 1994 and 1995. Values represent three pooled samples from riffles (R) or pools (P). Presence in qualitative samples given with $\alpha \mathrm{x} »$ is not included in the summations.

Tableau 3 (suite). Contenus des récoltes au filet des cours d'eau de Gran Canaria visités en 1994 et 1995. Les chiffres représentent trois récoltes de radiers $(R)$ et de mouilles $(P)$. La présence dans des récoltes qualitatives marquée par "X» n'est pas comptabilisée dans les totaux.

\begin{tabular}{|c|c|c|c|c|c|c|c|c|c|c|c|}
\hline \multirow[t]{3}{*}{$\overline{\text { Taxa }}$} & \multirow{3}{*}{$\begin{array}{l}\text { Siberio } \\
95 \\
P\end{array}$} & \multicolumn{2}{|c|}{ Sorta 1} & \multicolumn{2}{|c|}{ Soria 2} & \multicolumn{4}{|c|}{ Tejeda } & \multicolumn{2}{|c|}{ Tirajana } \\
\hline & & 94 & & 94 & & 94 & & 95 & & 94 & \\
\hline & & $\mathbf{R}$ & $P$ & $\mathbf{R}$ & $\mathbf{p}$ & A & $\mathbf{P}$ & $\mathbf{R}$ & $\mathbf{P}$ & A & $\mathbf{P}$ \\
\hline \multicolumn{12}{|l|}{$\overline{\text { Diptera }}$} \\
\hline Lmonildas indet & & 1 & 1 & & & 2 & & & & & \\
\hline Psychodidae indet. & & & & & & & 1 & & & & \\
\hline Dixa totrica & & & & 1 & & 1 & & & & 4 & 1 \\
\hline Anopheles sp. & 19 & & & & & & & 6 & 31 & & \\
\hline \multicolumn{12}{|l|}{ Culiseta longiareolata } \\
\hline Culex sp. & 3 & & & & & & & & 2 & & \\
\hline Simufium intermadium & & & & 95 & & 1 & & & & 172 & \\
\hline Simufium nficome & $x$ & & & & & & & 500 & & & \\
\hline Ceratopogonidae indet. & 10 & & & & & & 1 & & & & $x$ \\
\hline Ablabesmyia dusoleili & $x$ & & & & & & & 6 & 3 & & 6 \\
\hline Macropelopia nebulosa & & & & & & & 1 & & & & \\
\hline Macropelopia sp. & & & & & 1 & & 4 & & & 12 & \\
\hline Paramerina cingulata & & & & & & & 1 & & & & 5 \\
\hline Procladius choreus & $x$ & & & & & & & & & & \\
\hline Thienemannimyia sp. & & & & & & & & & & 1 & 1 \\
\hline Trissopelopla ?flavida & & & & & 2 & & & & & & \\
\hline Zavrelimyla barbatipes & & & & & 10 & & & & & & 3 \\
\hline Eukjefferlella brevicalcar/claripennis & & 3 & & & & 1 & & & & & \\
\hline Eukiefferialla minor & & 6 & & & & 2 & & & & 1 & \\
\hline Eukiefferella indet & & & & & & 3 & & & & & \\
\hline Heterotissocladius sp. & & & 4 & & & & & & & & \\
\hline Orthocladius (Eudactylocladius) fuscimanus & & 15 & & 1 & 1 & & & & & & \\
\hline Orthocladius (Euorthocladius) mivicola & & 5 & & 106 & 2 & 71 & 2 & & & 23 & \\
\hline Orthocladius (Orthocladius) pedestris & & 2 & & 4 & 221 & 3 & 12 & & & 77 & 44 \\
\hline Parametriocnemus styatus & $\mathbf{x}$ & & & & & & & 3 & & & \\
\hline Paratrichocladius sp. & & & & 4 & 13 & 42 & 64 & 5 & & 28 & 40 \\
\hline Psectrocladius & $x$ & & & & & & & & & & \\
\hline Rheocricotopus atipes & & & & & & 1 & & & & & \\
\hline Thienemanniella sp. nr clavicornis & & & & 4 & & & & 3 & & 8 & 1 \\
\hline Chironomus sp. & 3 & & & & & & & & & & \\
\hline Dicrotendipes sp & $x$ & & & & & & & & & & \\
\hline Phaenopsectra sp. & & & & & & & & & & & 4 \\
\hline Stictochironomus sp. & & & & & & & 1 & 1 & & & \\
\hline Rheotanytarsus sp. & $x$ & & & & & & & 1 & & & \\
\hline Empididae indet & & & & 1 & 6 & & 1 & 1 & & & 1 \\
\hline Dolichopodidae indet. & & & & & 1 & & & & & & \\
\hline Limnophora sp. & & & & & & & $x$ & & & & \\
\hline No. of inds. & 197 & 66 & 11 & 223 & 298 & 163 & 134 & 715 & 177 & 395 & 222 \\
\hline No. of taxa & 19 & $B$ & 6 & 12 & 17 & 16 & 25 & 21 & 17 & 17 & 24 \\
\hline
\end{tabular}

Belle (1982) collected it at Maspalomas in 1981. We collected larvae in stream pools at Carrizal 2 and Siberio.

Aeshnidae

Anax imperator Leach, 1815 WP

This species was reported from Sta Brigida by Valle (1935). In the period 1976-1986 Báez (unpubl.) recorded it from several additional localities : Bco. Fataga, Agaete, Tafira Alta, Las Palmas and Telde. Our only more recent record is from rock pools at Moya in 1995.

Anax parthenope Sélys, 1839 WP
Belle (1982) collected this species at Maspalomas, and Báez (unpubl.) recorded it from Bco. Fataga, Ingenio and Arguineguín between the years 1976-1986.

Hemianax ephippiger (Burmeister, 1839) Af

The only record from Gran Canaria we have seen is that of Belle (1982) from Maspalomas.

Libellulidae

Orthetrum chrysostigma (Bumeister, 1839) Af

This species was first recorded from Gran Canaria by Navas (1906; Teror), and later by Valle (1935) from Las Lagunetas 
Table 4. List of taxa found in qualitative samples from the localities or visits without quantitative sampling. Localities coded as: (Br) Brezal, (Ca1-2) Carrizal 1-2, (Co5) Coruña 1995, (Ga4-5) Gañanías 1994 \& 1995, (Gu4-5) Guayadeque 1994 \& 1995, (HA) Hondo de Abajo, (Ho) Hornos, (Mil) Mina 1, (Mo5) Moya 1995, (Pd) Peréz dam, (S34-35) Soria 31994 \& 1995, and (Ti5) Tirajana 1995.

Tableau 4. Liste des taxa trouvés dans des récoltes qualitatives de sites ou de prélèvements non quantitatifs. Codes des localités : (Br.) Brezal, (Ca 1-2) Carrizal 1-2, (Co5) Coruña 1995, (Ga 4-5) Gafianías 1994 \& 1995, (Gu 4-5) Guayadeque 1994 \& 1995, (HA) Hondo de Abajo, (Ho) Hornos, (Mil) Mina 1, (Mo5) Moya 1995, (Pd) Perez dam, (S 34-35) Soria 31994 \& 1995 et (Ti5) Tirajana 1995.

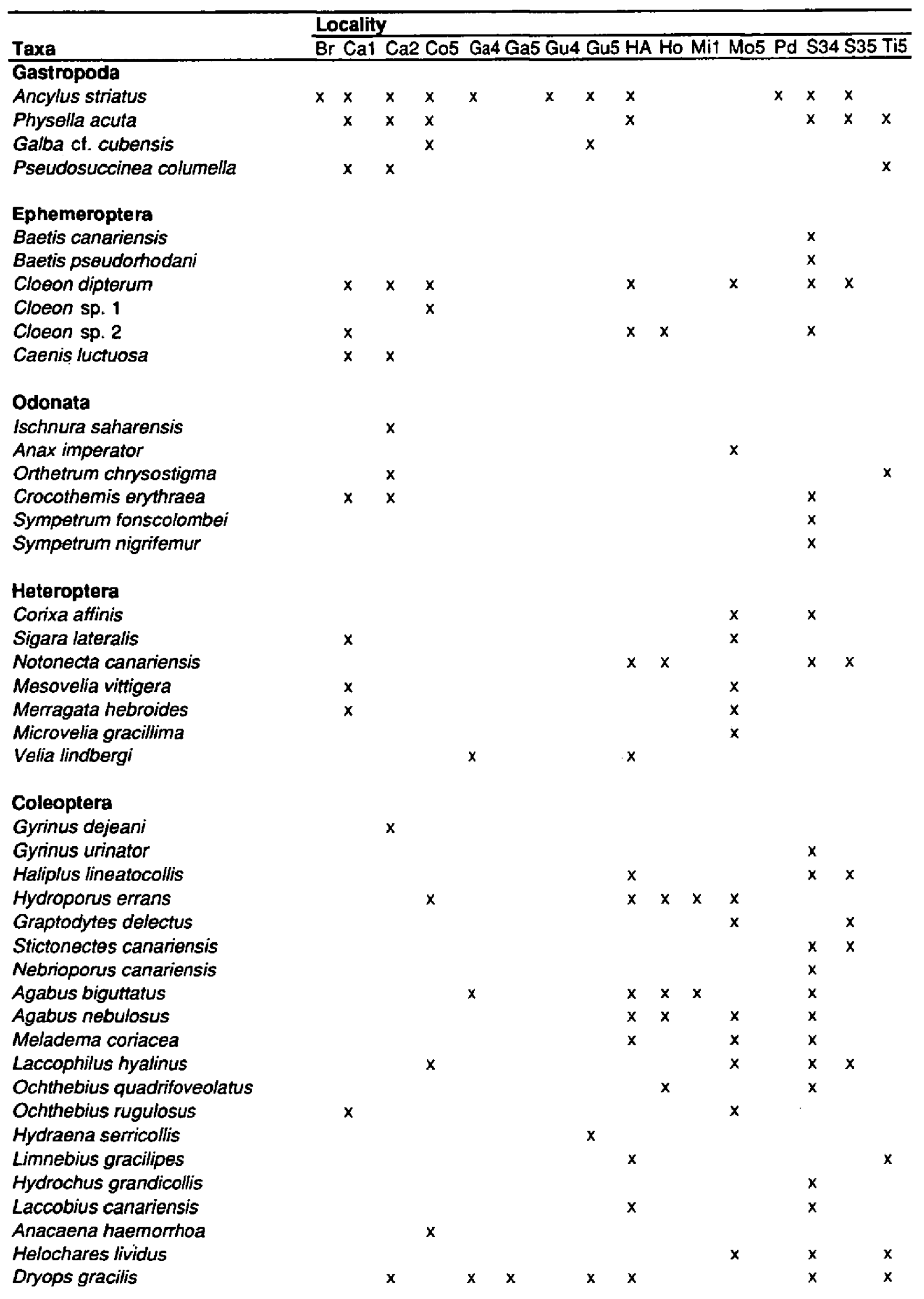


Table 4 (continued). List of taxa found in qualitative samples from the localities or visits without quantitative sampling. Localities coded as: (Br) Brezal, (Ca1-2) Carrizal 1-2, (Co5) Conuña 1995, (Ga4-5) Gañanías 1994 \& 1995, (Gu4-5) Guayadeque 1994 \& 1995, (HA) Hondo de Abajo, (Ho) Homos, (Mi1) Mina 1, (Mo5) Moya 1995, (Pd) Peréz dam, (S34-35) Soria 31994 \& 1995, and (Ti5) Tirajana 1995.

Tableau 4 (suite). Liste des taxa trouvés dans des récoltes qualitatives de sites ou de prélèvements non quantitatifs. Codes des localités : (Br.) Brezal, (Ca 1-2) Carrizal 1-2, (Co5) Coruña 1995, (Ga 4-5) Gañanías 1994 \& 1995, (Gu 4-5) Guayadeque 1994 \& 1995, (HA) Hondo de Abajo, (Ho) Hornos, (Mil) Mina 1, (Mo5) Moya 1995, (Pd) Perez dam, (S 34-35) Soria 31994 \& 1995 et (Ti5) Tirajana 1995.

\begin{tabular}{ll}
\hline Taxa & Locality \\
\hline Br Ca1 Ca2 Co5 Ga4 Ga5 Gu4 Gu5 HA Ho Mi1 Mo5 Pd S34 S35 Ti5 \\
\hline
\end{tabular}

Trichoptera

Hydroptila tortunata

Orthotrichia angustella

Oxyethira sp.

Stactobia storai

Tinodes canariensis

Hydropsyche maroccana

Mesophylax aspersus

\section{Diptera}

Limoniidae indet.

Psychodidae indet.

Dixa tetrica

Anopheles sp.

Culiseta longiareolata

Culex sp.

Thaumalea subafricana

Simulium guimari

Simulium intermedium

Simulium paraloutetense

Simulium ruficorne

Ceratopogonidae indet.

Ablabesmyia dusoleili

Macropelopia sp.

Procladius choreus

Zavrelimyia barbatipes

Chaetocladius sp.

Cricotopus (Isocladius) sp.

Eukiefferiella brevicalcar/clanipennis

Eukiefferiella minor

Orthocladius (Eud.) fuscimanus

Orthocladius (Euo.) rivicola

Orthocladius (O.) pedestris

Parametriocnemus stylatus

Paratrichocladius sp.

Psectrocladius sp.

Rheocricotopus atripes

Thienemanniella sp. nr clavicornis

Chironomus sp.

Dicrotendipes sp.

Phaenopsectra sp.

Cladotanytarsus sp.

Micropsectra sp.

Rheotanytarsus sp.

Virgatanytarsus sp.

Tanytarsini indet.

Oxycera grancanariensis

Dolichopodidae indet.

Limnophora sp.
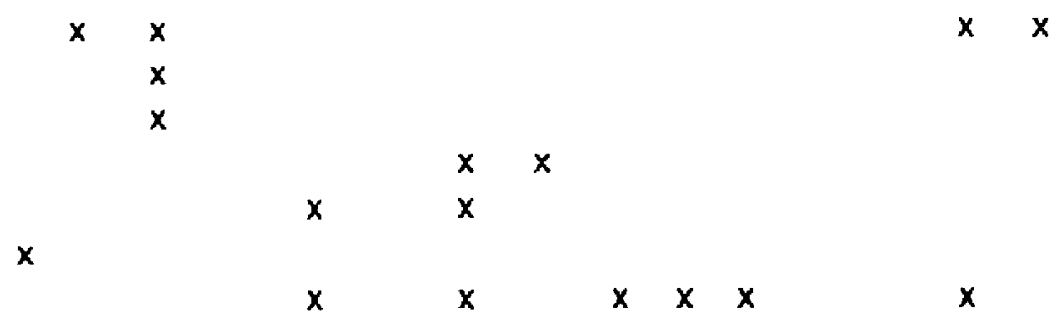

x

$x$

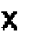

$x$

$x \quad x$

$x$

$x$

$\mathbf{x}$

$x$

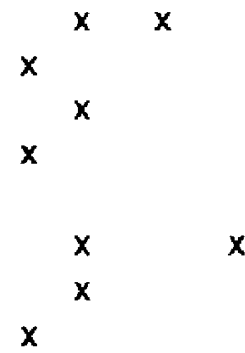

$x \quad x$

$x \quad x$

$x$

$x$

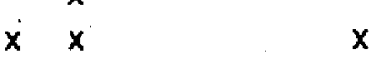

$x \times x x$

$x \quad x$

$x \times x$

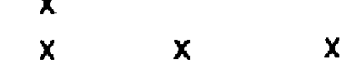

X $\mathrm{x}$

$\mathrm{x} x$

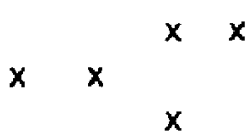

No. of taxa

$\begin{array}{llllllllllllllll}8 & 21 & 21 & 12 & 13 & 3 & 9 & 12 & 31 & 20 & 7 & 17 & 3 & 37 & 12 & 11\end{array}$ 
and Tafira, and by Gardner (1960) from Maspalomas. Besides our three records in Table 3, we also collected it at Carrizal 2 and Tirajana in 1995.

Crocothemis erythraea (Brullé, 1832) Me

Valle $(1935,1955)$ recorded this species from Moya and Aldea San Nicolás, and it was later recorded from Maspalomas by Gardner (1960) and Belle (1982). Báez (unpubl.) recorded it from eight additional sites between 1976 and 1986. We collected it at : Carrizal 1-2, Siberio, Soria 3, and Tejeda.

Sympetrum fonscolombei (Sélys, 1840) Me

Eaton collected this species at Las Palmas already in 1880 (McLachlan 1883), and later Valle (1935) recorded it from Tafira, and Gardner (1960) from Maspalomas. Báez (unpubl.) collected it at Moya and Tafira Alta some time between 1976 and 1986. We collected larvae only at Azuaje and Soria 3.

Sympetrum nigrifemur (Sélys, 1884) Ma

Valle (1935) reported on specimens collected at Moya in 1935. Our only additional record is from isolated stream pools at Soria 3.

Trithemis arteriosa (Burmeister, 1839) Af

According to Brauer (1901), this species was collected at San Mateo in 1890. Gardner (1960) later added a more recent record from Maspalomas. Báez (unpubl.) recorded it from five additional sites 1976-1986. We found it in stream pools only at Moya and Siberio.

Zygonyx torrida (Kirby, 1889) Af

The only published records of this species from Gran Canaria are those of Brauer (1901), who examined specimens collected in 1890 at Roque del Nublo and Cumbre altiplano 900$1100 \mathrm{~m}$. Our only record is from riffles at Azuaje in 1995.

\subsection{Hemiptera}

Corixidae

Corixa affinis Leach, 1817 WP

This species was collected at Aldea San Nicolás and Maspalomas in 1949 (Lindberg 1953), and at Tejeda in 1981 (Baena \& Báez 1990). We found it at the following four localities : Coruña, Moya, Siberio and Soria 3.

Sigara hoggarica Poisson, 1929 Af

The only Gran Canarian record of this species is from between Mogan and San Nicolás (Baena \& Báez 1990).

Sigara lateralis (Leach, 1817) WP

This species was collected at Maspalomas in 1949 (Lindberg 1953), and later also at Bco. de Tejeda in 1981 (Baena \& Báez 1990). We collected it at Carrizal 1, Moya and Siberio.

Sigara selecta (Fieber, 1848) WP

This species is in Gran Canaria known only from Maspalomas, where it was collected in 1954 and 1962 (Baena \& Báez 1990).

Heliocorisa vermiculata (Puton, 1874) $\mathrm{Me}$

This species was recorded from Gran Canaria by Baena \& Báez (1990), without any detailed information.

\section{Notonectidae}

Notonecta canariensis Kirkaldy, 1897 Ca (G T)

Baena \& Báez (1990) gave records from Moya and Tejeda from the early 1980 'ties. Besides the seven localities listed in Table 3, we found it also at: Hondo de Abajo, Hornos, and Soria 3.

Anisops debilis canariensis (Noualhier, 1893) Af (Ca)

The only published record we have seen is from Aldea San Nicolás in 1949 (Lindberg 1953).

Anisops sardeus Herrich-Schäffer, $1849 \mathrm{Me}$

Baena \& Báez (1990) listed this species for Gran Canaria without any more detailed information.

Mesoveliidae

Mesovelia vittigera Horvath, 1895 Af

Our 1995 records of this species from stream pools at Carrizal 1 and Moya are seemingly the first ones from Gran Canaria.

Hebridae

Hebrus pusillus (Fallén, 1807) WP

Lindberg (1953) reported on this species from Azuaje in 1927 and Aldea San Nicolás and Valle de Tejeda in 1949. It was refound at the last locality in 1973 according to Baena \& Báez (1990), and in 1994 by us. We can also add a record from Siberio in 1995.

Merragata hebroides Buchanan-White, 1877 Am

This species was collected at Aldea San Nicolás in 1949 by Lindberg (1953). We found it in 1995 at Carrizal 1 and Moya.

Hydrometridae

Hydrometra stagnorum (Linnaeus, 1758) WP

Lindberg (1953) recorded this species from the following five localities : Aldea San Nicolás, Arucas, Las Lagunetas, Moya, and Valle de Tejeda. It was refound at the last locality in 1977 according to Baena \& Báez (1990). Our only record is from Cernicalos, where it was found both in 1994 and 1995.

Veliidae

Microvelia gracillima Reuter, 1883 Af

Our records from Azuaje, Moya, Siberio and Tejeda are apparently the first ones from Gran Canaria.

Velia lindbergi Tamanini, $1954 \mathrm{Ca}$ (G LP T)

In the original description of this species, Tamanini (1954) gave records from Las Lagunetas and Tejeda from 1947. We found it abundantly at Cernicalos and present also at Gañanías, Hondo de Abajo, and Mina 3 (Tables 3-4).

Gerridae

Gerris thoracicus Schummel, 1832 WP

Zimmermann (1984) and Baena \& Báez (1990) recorded this species from Gran Canaria without giving any more detailed information on localities. 


\subsection{Coleoptera}

\section{Gyrinidae}

Gyrinus dejeani Brullé, $1832 \mathrm{Me}$

Wollaston (1864) gave a record from Arguineguin, and more recently Balke et al. (1990) added records from six localities. Besides the eight localities given in Table 3, we found it also at Carrizal 2.

\section{Gyrinus urinator Illiger, 1807 WP}

This species was recorded from Gran Canaria already by Wollaston (1864). Six more recent records were added by Balke et al. (1990). Besides the two localities given in Table 3 , we found it also at Soria 3 .

Aulonogyrus striatus (Fabricius, 1792) Me

Wollaston (1864) recorded this species from El Charco at Maspalomas, where it was «tolerably common ..., though somewhat local». As it has never again been found in Gran Canaria, it is seemingly extinct on this island.

Haliplidae

\section{Haliplus lineatocollis (Marsham, 1802) WP}

First recorded from Gran Canaria at El Monte in 1858 by Wollaston (1864). More recently Balke et al. (1990) recorded it from eight additional localities. Besides the seven localities listed in Table 3, we found it also at Hondo de Abajo and Soria 3.

\section{Dytiscidae}

\section{Bidessus minutissimus (Germar, 1824) WP}

Wollaston (1864) recorded this species from El Monte in 1858. It was later collected at Tejeda in 1949 and Bco. del Sauce in 1957 (Machado 1987b). More recently Balke et al. (1990) collected it in stream pools in Bco. de Tirajana at $1000 \mathrm{~m}$ asl. We found it still abundant in the same pools, and added also records from Azuaje, Siberio, Soria 2, and Tejeda. Hygrotus confluens (Fabricius, 1787) WP

This species was collected at Maspalomas in 1950 and in three more ponds or reservoirs in the mid 1980'ties (Machado 1987b). Balke et al. (1990) added a record from Tamaraceite, Piletas. The absence of this species from our samples is probably due to its preference for larger ponds; a habitat not sampled by us.

Herophydrus musicus (Klug, 1833) Me

This species was recorded from Gran Canaria already by Wollaston (1864). Later, Machado (1987b) could add four localities and Balke et al. (1990) three. We collected it at Azuaje and Moya in 1994.

\section{Hyphydrus maculatus Babington, 1841 Af}

This species was collected at Azuaje and Santa Brigida in 1927, and more recently in Bco. de Fataga in 1973 (Machado $1987 \mathrm{~b}$; as $H$. crassus). More recently it was found also in Bco. de Tirajana near San Bartolomé (Balke et al. 1990). Our only specimens were found in stream pools at Moya in 1994.

Hydrotarsus pilosus Guignot, $1945 \mathrm{Ca}$ (T)
This species was first found in Gran Canaria at Teror in 1935 and at Artenara in 1957 (Machado 1987b). More recently, Balke et al. (1990) found it in seeping water $2 \mathrm{~km} \mathrm{~W}$ Ayacata and at Pico de las Nieves at altitudes of 1200-1700m (Balke et al. 1990). Our only specimen was found at Moya in 1994, at $290 \mathrm{~m}$ asl.

\section{Hydroporus errans Sharp, 1882 Ca (G H LP T)}

This species is very common in Gran Canaria. Machado (1987b) listed eight localities where it had been found between 1950 and 1984, and Balke et al. (1990) added ten more recent records. Besides the nine localities listed in Table 3, we found it also at Hondo de Abajo, Hornos, and Mina 1.

Hydroporus lucasi Reiche, $1866 \mathrm{Me}$

Machado (1987b) listed this species from Las Lagunetas, San Bartolomé and Teror 1971-1973. Balke et al. (1990) gave a more recent record from the last locality. We did not find it in Gran Canaria.

Graptodytes delectus (Wollaston, 1864) Ca (T)

The oldest Gran Canarian record of this species is from 1927 in Bco. Azuaje (Machado 1987b). During the 80ties it was found at eight additional localities (Machado 1987b, Balke et al. 1990). We can add the following localities : Moya, Siberio, Soria 2-3, and Tejeda.

Stictonectes canariensis Machado, 1987 GC

In Gran Canaria, this species was first collected at Teror in 1971 (Machado 1987b). Nine more recent records were listed by Machado (1987b) and Balke et al (1990). Besides the six localities listed in Table 3, we can add also Soria 3. The larva of this species was recently described by Alarie \& Nilsson (1997).

\section{Nebrioporus canariensis (Bedel, 1881) Ca (G H LP T)}

According to Wollaston (1864; as tessellatus) this was once "An abundant insect in nearly all the streams of Gran Canary». More recent records are, however, quite few. Machado (1987b) recorded it from San Bartolomé in 1955 and Azuaje in 1963. It was refound at the former locality by Balke et al. (1990). All our records are from 1994, when we found it at Cernicalos, Mina 3 and Soria 3 (Tables 3-4).

Agabus biguttatus (Olivier, 1795) WP

Wollaston (1864) recorded this species from $\mathrm{El}$ Monte in 1858. Machado (1987b) added eight records from the last 50 years, and Balke et al. (1990) could add 11 more recent ones. Besides the seven localities listed in Table 3, we can add also Gañanías, Hondo de Abajo, Hornos, Mina 1, and Soria 3. Agabus conspersus (Marsham, 1802) WP

Machado (1987b) confirmed the old Lindberg records of this species from Maspalomas in 1950 and Valle de Tejeda in 1949. As no more recent records are known it might well be considered extinct from Gran Canaria. The absence of this species in our samples may also have been caused by the fact that we did not sample any low-altitude ponds.

Agabus nebulosus (Forster, 1771) WP

In Gran Canaria, this species was first recorded from Tafira by Wollaston (1864). Sixteen more recent records were given 
by (Machado 1987b) and (Balke et al. 1990). Besides the four localities listed in Table 3, we found it also at Hondo de Abajo, Hornos, and Soria 3.

\section{Meladema coriacea Laporte, $1835 \mathrm{Me}$}

Wollaston (1864) recorded this species from El Charco in Maspalomas. Machado (1987b) added records from Tejeda in 1973 and 1981, and Balke et al. (1990) gave more recent records from Artenara, San Bartolomé, La Culata and Teror. Besides the five localities given in Table 3, we found this species at Hondo de Abajo, and Soria 3.

\section{Laccophilus hyalinus (De Geer, 1774) WP}

Wollaston (1864) knew three Gran Canarian localities for this species. Machado (1987b) added three records spanning from 1949 to 1973, and Balke et al. (1990) gave five more recent records. We found it abundant at Azuaje, Moya and Tejeda (Table 3), and present also at Coruña, Siberio, and Soria 3.

Eretes sticticus (Linnaeus, 1758) Co

This species was collected in El Charco at Maspalomas first in 1858 (Wollaston 1864), and most recently in 1950 (Machado 1987b). Most probably it is now extinct in Gran Canaria.

\section{Cybister tripunctatus (Olivier, 1795) Af}

Wollaston (1864) knew this species from Arguineguin, where in 1858 he found it «in the pools, or small freshwater lakes, close to the sea». It was later recorded from Aldea San Nicolás in 1949 and from Maspalomas in 1950 (Machado 1987b), and more recently from Tamaraceite at Piletas (Balke et al. 1990). We have not observed it in Gran Canaria; most probably because we did not sample any low-altitude ponds.

\section{Hydraenidae}

\section{Ochthebius lapidicola Wollaston, 1864 Ca (G H LP T)}

Orchymont (1940) found this species in Azuaje at $300 \mathrm{~m}$ asl in 1935. More recently Balke et al. (1990) recorded it from wet rocks at $1200-1500 \mathrm{~m}$ on Pico de las Nieves.

Ochthebius quadrifoveolatus Wollaston, $1854 \mathrm{Me}$

Wollaston (1864) knew this species from El Monte in Gran Canaria. Orchymont (1940) found it common during his 1935 visit, and listed seven localities ranging in altitude from 300 to $1285 \mathrm{~m}$ asl. More recently, Balke et al. (1990) added four more localities at relatively high altitudes. Besides the three localities listed in Table 3, we found this species at Hornos and Soria 3.

[Ochthebius quadricollis Mulsant, $1844 \mathrm{Me}$

Orchymont (1940) recorded this species from Puerto de la Luz. As it lives in marine rock pools, it is not strictly limnic.] Ochthebius rugulosus Wollaston, $1857 \mathrm{Ma}$

The oldest record of this species from Gran Canaria appears to be that from Atalaya mentioned by Orchymont (1940). More recently, Balke et al. (1990) listed seven additional localities. We collected it at the following sites : Azuaje, Carrizal 1, Cernicalos, Moya, Soria 1, and Tirajana.

\section{Hydraena serricollis Wollaston, 1864 Ca (G LP T)}

This is seemingly a rare species in Gran Canaria. It was recorded from Bco. de Tirajana at San Bartolomé by Balke et al. (1990). We collected it at the same locality, and also at Guayadeque and Siberio.

\section{Limnebius canariensis Orchymont, 1938 GC}

This rare species is endemic to Gran Canaria. The holotype was collected at $1000 \mathrm{~m}$ asl in Bco. de Tirajana (Orchymont 1940). Jäch (1993) recorded a second male from the same valley at $1200 \mathrm{~m}$ asl, collected in 1989 . We collected a single male at Soria 2 in 1994.

\section{Limnebius gracilipes Wollaston, 1864 Ca (G LP T)}

Wollaston (1864) described this species from a stream at Mogan in Gran Canaria. Orchymont (1940) listed three additional localities, and Jäch (1993) gave four more sites in his revision of this genus. We collected this species at the following seven localities : Cernicalos, Coruña, Hondo de Abajo, Siberio, Soria 2, Tejeda and Tirajana (Tabs 3-4).

Hydrochidae

Hydrochus grandicollis Kiesenwetter, $1870 \mathrm{Me}$

Balke et al. (1990) found this species in pools at La Culata. We collected it at the following five localities : Mina 3, Siberio, Soria 2-3, and Tejeda (Tables 3-4).

Hydrophilidae

Coelostoma hispanicum (Küster, 1848) Me

This species was first reported from Gran Canaria by Wollaston (1864; as C. orbiculare). Orchymont (1940) and Balke et al. (1990) each added two more localities. We found it at Azuaje, Siberio and Tejeda.

\section{Chaetarthria similis Wollaston, 1864 WP}

Wollaston (1864) knew this species from El Monte in Gran Canaria, and Orchymont (1940) later found it in Bco. Azuaje. More recently, Balke et al. (1990) recorded it from San Bartolomé and Teror. We found it only at Tejeda in 1994.

Laccobius canariensis Orchymont, 1940 Ca (F G L LP T)

This is seemingly the most common hydrophilid species in Gran Canaria, from where it was recorded already by Wollaston (1864; as L. minutus). It was Orchymont (1940) who first recognized it as a Canarian endemic, of which the type locality is Los Tilos in Gran Canaria. He listed it also from five other localities, and more recently Balke et al. (1990) added nine more. Besides the five localities listed in Table 3, we found it at Hondo de Abajo, and Soria 3.

Laccobius gracilis Motschulsky, $1855 \mathrm{Me}$

This is seemingly a recent immigrant to the Canary Islands. Balke et al. (1990) found it in Gran Canaria in Bco. de Tirajana at San Bartolomé, and in Bco. Tederas at Las Tederas. Our only record is from Tejeda in 1994.

\section{Anacaena haemorrhoa (Wollaston, 1864) Ca (G T)}

Wollaston (1864) first described this species from Teror and Taraja in Gran Canaria, where it was found «about wet rocks». Later Orchymont (1940) collected it at Teror and listed a record from Las Lagunetas. We found it abundant at Cernicalos, and present also at Azuaje and Coruña (Tables 34). 


\section{Helochares lividus (Forster, 1771) WP}

Balke et al. (1990) listed this species from seven localities in Gran Canaria. We collected it at the following seven localities : Azuaje, Moya, Siberio, Soria 2-3, Tejeda, and Tirajana (Tables 3-4).

Enochrus politus (Küster, 1849) Me

This species was first recorded from Gran Canaria by Wollaston (1864; as Philhydrus melanocephalus). Later, Orchymont (1940) added three more localities. More recently, Balke et al. (1990) found it only at Teror.

Enochrus bicolor (Fabricius, 1792) WP

The only Gran Canarian record of this species appears to be that of Orchymont (1940) from Maspalomas.

\section{Dryopidae}

\section{Dryops gracilis Karsch, $1881 \mathrm{Me}$}

This species was first listed from Gran Canaria by Wollaston (1864; as proliferatus). Balke et al. (1990) found it at all their localities on this island, and it was present also at the great majority of our localities (Tables 3-4).

\section{Scirtidae}

\section{Cyphon gracilicomis Wollaston, $1864 \mathrm{Ca}$ (G T)}

This species was first described from El Monte in Gran Canaria. It is absent from our material.

\subsection{Trichoptera}

Glossosomatidae

Agapetus adejensis Enderlein, $1929 \mathrm{Ca}$ (G LP T)

The only published record of this species from Gran Canaria is that of Nybom (1948) from Las Lagunetas at $800 \mathrm{~m}$ asl. Larvae of this species were relatively common at Cernicalos, and found nowhere else.

Hydroptilidae

Hydroptila fortunata Morton, $1893 \mathrm{Ma}$

Nybom (1948) listed Eaton's old records of this species from San Mateo and Teror, and added also a newer one from Las Lagunetas at $800 \mathrm{~m}$ asl. We collected adults of this species in our light traps at Azuaje, Mina 2 and Tirajana, and found also some adults at Moya and Soria 3. Besides the seven localities listed in Table 3, we found larvae also at Carrizal 1-2 and Soria 3.

\section{Orthotrichia angustella McLachlan, $1865 \mathrm{Me}$}

Adults of both sexes of this species were collected in our light traps at Azuaje both in 1994 and 1995. Besides at Azuaje, larvae were also found at Carrizal 2 in 1995. Our records are seemingly the first ones from Gran Canaria.

\section{Oxyethira spinosella McLachlan, $1883 \mathrm{Ma}$}

The only published Gran Canarian record of this species is that of Nybom (1948). This record is, however, ambiguous as the given locality «Hermigua, Barrancos» is situated in La Gomera. We collected one male in a light trap at Tirajana in 1995. Oxyethira larvae were found at Azuaje, Carrizal 2, Moya, and Siberio (Tables 3-4).

\section{Oxyethira falcata Morton, 1893 WP}

Our light traps collected one male of this species at Azuaje on 31 March 1994 and one female at Tirajana on 2 January 1995. Some or all of the larval records given above may refer to this species.

Stactobia freyi Nybom, 1948 GC

This species was described from three males collected at a damp cliff near Las Lagunetas at $800 \mathrm{~m}$ asl in 1931 (Nybom 1948). The specimens collected by Eaton at Teror in 1880 listed by McLachlan (1883) as $S$. atra may belong to this or the next species.

Stactobia storai Nybom, 1948 Ca (G LP T)

We collected Stactobia larvae at Guayadeque both in 1994 and 1995 . The only adult we found there was identified as $S$. storai, though some minor deviations from material from Tenerife indicate that more specimens are needed for certain identification (Malicky, in litt.).

Psychomyiidae

Tinodes canariensis McLachlan, $1883 \mathrm{Ca}$ (G LP T)

This species was first described from specimens collected by Eaton at San Mateo in 1880 (McLachlan 1883). Later, Nybom (1948) added a record from Las Lagunetas at $800 \mathrm{~m}$ asl. We collected two males and four females in a light trap at Mina 2 on 19 November 1995. Moreover, one male pupa collected at Guayadeque together with larvae was identified as T. canariensis. The larvae collected at Gañanias may belong to this or to the next species.

Tinodes arcuata Nybom, 1948 GC

This species was described from a single male collected at a mountain brook at Las Lagunetas in 1931 (Nybom 1948). We know of no subsequent records.

Polycentropodidae

Polycentropus sp.

Larvae belonging to this genus were collected at Tirajana in 1994 and at Siberio in 1995. Most likely they belong to the same, still unidentified, species as the larvae collected in Tenerife (Malmqvist et al. 1995).

Hydrosychidae

Hydropsyche maderensis Hagen, $1865 \mathrm{Ma}$

Nybom (1948) reported on three males of this species collected at Las Lagunetas in 1931. We know of no subsequent records of this otherwise Madeiran species.

Hydropsyche maroccana Navas, 1935 Af

This species was recorded from a small stream at Las Lagunetas by Nybom (1948; as $H$. flava). We collected adults in our light traps at Azuaje and Tirajana in 1995. Larvae of this genus were abundant at Cernicalos and Mina 2, and present also at Azuaje, Mina 3, and in a canal at Brezal.

Limnephilidae

Mesophylax aspersus Rambur, 1842 WP

This species was first collected in Gran Canaria at San Mateo by Eaton in 1880 (McLachlan 1883). Later, Nybom (1954) 
gave records from Tamadabe in 1949 and Cruz de Tejeda in 1950. We collected adults in light traps at Mina 2, Soria 1 and Tirajana. Larvae were abundant at several of the localities listed in Table 3, and present also at Gañanías, Guayadeque, Hondo de Abajo, Hornos, Mina 1, and Soria 3.

Leptoceridae

Oecetis canariensis Brauer, $1900 \mathrm{Ca}$ (T)

This species was first described from Bco. de los Chorros, above San Mateo, at $790 \mathrm{~m}$ asl in 1890 (Brauer 1901). We know of no subsequent records from Gran Canaria.

\subsection{Diptera}

Limoniidae

[The treatment of this family chiefly follows Theowald (1977) and Jong (1987).]

Dicranomyia chorea (Meigen, 1818) WP

This species was first reported from Gran Canaria by Lackschewitz (1936), who listed it from a stream at Las Lagunetas. Later, Hemmingsen et al. (1959) added Bco. Bandama, Los Laureles, and Valle Angostura. We collected adults in our light traps at Azuaje and Mina 2. Larvae of this genus were found at four of the localities in Table 3, and also at Brezal, Gañanías, Guayadeque, Hondo de Abajo, and Mina 1.

Dicranomyia hamata Becker, $1908 \mathrm{Me}$

Hemmingsen et al. (1959) found this species at seven localities in Gran Canaria. It was not found in our samples.

Dicranomyia intermedia Santos, 1923 Ca (G T LP)

We collected adults of this species in our light traps at Azuaje, Mina 2, and Tirajana in 1995. It was recorded from Gran Canaria by Theowald (1977).

- Dicranomyia vicina (Macquart, 1838) Ma

This species was first recorded from Gran Canaria by Lackschewitz (1936; as canariensis) from a stream at Las Lagunetas. Later, Hemmingsen et al. (1959; as canariensis) recorded it from Bco. Bandama, Bco. los Gatos and Bco. la Higuera. We collected adults of this species at Azuaje, Mina 12 , Soria 1 , and Tirajana.

[Geranomyia atlantica annulirostris Pierre, $1918 \mathrm{Ma}$

Lackschewitz (1936; as cabrerae) gave a record of this species from a pool near the sea at Puerto de la Luz. The habitat may thus not be strictly limnic.]

Geranomyia canariensis Bergroth, $1889 \mathrm{Ma}$

Lackschewitz (1936; as maculipennis) reported this species from Las Lagunetas. We know of no subsequent records from Gran Canaria.

[Geranomyia unicolor (Haliday, 1833) Ca (L T)

Lackschewitz (1936; as umbratica) gave a record of this species from a pool near the sea at Puerto de la Luz. The habitat may thus not be strictly limnic.]

Psychodidae

[As older records of this family from Gran Canaria have not been revised, we list only our own ones. Tonnoir (1921) re- corded Clogmia albipunctata and Tinearia alternata from Gran Canaria, but the other species on our list are here seemingly recorded from the island for the first time.]

Satchelliella binunciolata (Satchell, 1955) Ca (LP T)

We collected manually 11 adult specimens at Guayadeque and one specimen at Tejeda in 1994. A single larva was found at Mina 1.

Satchelliella tenerifensis (Satchell, 1955) Ca (LP T)

We collected one larva at Azuaje and two at Mina 3 in 1994.

Paramormia ustulata (Walker, 1856) Me

Adults of this species were collected in the light trap at Azuaje in 1994 and 1995.

Mormia tenebrosa (Satchell, 1955) Ca (LP T)

Adults of this species were collected in the light trap at Azuaje in 1994 and 1995.

Clogmia albipunctata (Williston, 1893) Co

Two males of this species were collected in the light trap at Azuaje in 1995.

Psychoda sp.

An unidentified Psychoda species was collected in the light trap at Azuaje in 1995, and also hand-collected at Guayadeque in 1994.

Tinearia alternata (Say, 1824) Co

Adults of this species were collected in light traps at Azuaje and Mina 2.

Tinearia lativentris (Berdén, 1952) Me

This species was found only in the light trap at Azuaje in 1995.

Philosepedon sp. humeralis-gr.

Single specimens of this species were found in the light trap at Azuaje in 1994 and 1995.

Dixidae

Dixa tetrica Peus, 1934 Ca (G LP T)

Besides the eight localities listed in Table 3, we found larvae of this species also at Gañanías, Guayadeque, Hondo de Abajo, Hornos, and Soria 3 . We are not aware of any previously published records from Gran Canaria.

Culicidae

The mosquito fauna of the Canary Islands was reviewed by Báez and Fernandez (1980). Eight species are known from Gran Canaria. We have collected only occasional adults, and larvae of the genera Anopheles, Culiseta, and Culex were found in some of the streams (Tables 3).

Thaumaleidae

Thaumalea subafricana (Becker, 1908) Ca (LP T)

Storå (1936) recorded this species from a wet rock near a waterfall at Las Lagunetas. We collected larvae at Guayadeque, Hondo de Abajao, and Mina 2. 


\section{Simuliidae}

[The earlier records of this family from Gran Canaria were revised by Crosskey (1988).]

Simulium guimari Becker, 1908 Ca (LP T)

This species was collected at Las Lagunetas in 1931. We found larvae abundant at Cernicalos and Mina 2. They were also present at Gañanías, Guayadeque, Mina $1 \& 3$, and Moya (Tables 3-4).

\section{Simulium intermedium Roubaud, 1906 WP}

This species was collected at Las Lagunetas and Tafira in 1931. Besides at the eight localities listed in Table 3, we found this species also at Brezal, Gañanías, Hondo de Abajo, Hornos, Mina 1, and Soria 3 (Table 4).

Simulium paraloutetense Crosskey, 1988 GC

This species was collected at Las Lagunetas in 1931, and later Crosskey (1988) suggested that it had became extinct. We found larvae and pupae at Guayadeque both in 1994 and 1995.

\section{Simulium pseudequinum Séguy, 1921 WP}

Previous records of this species from Gran Canaria include one without more detailed locality from 1890 , Bco. Guiniguada and Tafira 1903, and Las Lagunetas, Las Palmas and Tafira 1931. We found the larvae of this species abundantly at Azuaje in 1994. They were seemingly not present there in 1995 , and not found at any other of our localities.

\section{Simulium ruficome Macquart, 1838 Af}

This species was collected at Moya in 1931. We found it abundantly at Azuaje in both years, at Moya in 1994, and at Tejeda in 1995. Occasional specimens were found also at Brezal, Carrizal 1-2, and Siberio.

Ceratopogonidae

As our limited material of this family has not been identified to species and no recent revision is available we have refrained from dealing with this family. Larvae or pupae were found at five of our localities (Tables 3-4).

Chironomidae

[As most of the older records of this family from Gran Canaria have not been revised, we list only our own ones. Distribution codes refer to the geographic area from which most records originate.]

Tanypodinae

Ablabesmyia dusoleili Goethghebuer, 1935 Af

Adults of this species were collected in light traps at Azuaje and Tirajana. Besides the six localities given in Table 3 , larvae were also found at Carrizal 2.

\section{Macropelopia nebulosa (Meigen, 1804) WP}

Larvae of this species were found at Coruña and Tejeda. More larvae of this genus, not identified to species, occurred at Hondo de Abajo, Hornos, Mina 3, Soria 2, and Tirajana.

Paramerina cingulata (Walker, 1856) WP

Adults of this species were collected in light traps at Azuaje and Tirajana. Larvae were found at the five localities given in Table 3 .

\section{Procladius choreus (Meigen, 1804) Af \& WP}

Adults of this species were collected in light traps at Azuaje, Mina 2 and Tirajana. Larvae were found at Mina 3, Moya, Siberio, and Soria 3.

Thienemannimyia sp.

Larvae belonging to this genus were only found at Cernicalos and Tirajana (Table 3).

Trissopelopia ?flavida (Kieffer, 1923) WP

Larvae probably representing this species were only found at Soria 2 (Table 3).

Zavrelimyia barbatipes (Kieffer, 1911) WP

Adults of this species were collected in the light traps at Azuaje and Tirajana in 1995. Besides the seven localities given in Table 3, larvae occurred also at Hondo de Abajo, Hornos, and Soria 3.

Orthocladiinae

Bryophaenocladius cuneiformis Armitage, $1987 \mathrm{Ca}$ (T)

This species was previously known only from Tenerife. We collected one specimen in a light trap at Tirajana in November 1995.

Cardiocladius sp.

Larvae representing an unidentified species of this genus were found at Cernicalos and Mina 2 in 1995.

\section{Chaetocladius sp.}

Larvae representing an unidentified species of this genus were found at Gañanías and Mina 3 in 1994.

Corynoneura ?lobata Edwards, 1924 WP

Adults of this species were collected in the light trap at Mina 2 in 1995. Larvae of this genus were found at Coruna and Mina 2-3 (Table 3).

Cricotopus (Cricotopus) triannulatus (Macquart, 1826) WP

Adults of this species were found only on the wet rock at the Peréz dam.

Cricotopus (Cricotopus) vierriensis Goetghebuer, 1935 WP

Adults of this species were collected in the light traps at Azuaje and Tirajana in November 1995.

Cricotopus (Isocladius) sp.

Adults of this unidentified species were collected in the light traps at Azuaje and Mina 2 in 1995. Larvae of the same subgenus occurred only at Soria 3.

Eukiefferiella brevicalcar (Kieffer, 1911) or claripennis (Lundbeck 1898) WP

Adults of one of these two species were found in the light traps at Mina 2 and Soria 1. Larvae assigned to this speciespair were found at Hondo de Abajo, Soria 1 and Tejeda.

Eukiefferiella minor (Edwards, 1929) WP

Besides at the five localities given in Table 3, larvae of this species were also found at Gañanías and Hondo de Abajo.

Eukiefferiella sp. nr ilkleyensis (Edwards, 1929) WP

Larvae of this species were found only at Azuaje and Mina 2. 
Heterotrissocladius sp.

Larvae representing an unidentified species of this genus were found only at Soria 1 (Table 3).

Limnophyes sp.

Adults of one or more unidentified species of this genus were found in light traps at Azuaje, Mina 2, and Tirajana, and hand-collected at Soria 3. Larvae were found only at Mina 3 and Moya (Table 3).

Orthocladius (Eudactylocladius) fuscimanus (Kieffer, 1908) WP

Our light traps collected at least six unidentified species belonging to this genus. Besides at the three localities given in Table 3, larvae of this species were found also at Hornos.

Orthocladius (Euorthocladius) rivicola Kieffer, 1911 WP

Besides at the six localities given in Table 3, larvae of this species were found also at Hondo de Abajo, Hornos, and Soria 3.

Orthocladius (Orthocladius) pedestris (Kieffer, 1909) WP

Larvae identified as $O$. pedestris occurred at the seven localities given in Table 3, plus Hondo de Abajo, Hornos, Peréz dam, and Soria 3.

Parametriocnemus stylatus (Kieffer, 1924) WP

Adults of this species were found only at Azuaje in 1994, but in 1995 they were found also at Carrizal 2, Guayadeque, Mina 2 , and Tirajana. Besides at the seven localities given in Tab. 3, larvae of this species were found also at Carrizal 1-2, and Hondo de Abajo.

Paratrichocladius rufiventris (Meigen, 1830) WP

Adults of this species occurred in the 1994 adult samples from Azuaje, Soria 3, Tejeda, and Tirajana. Larvae probably representing rufiventris plus one unidentified species occurred at eight of the localities given in Table 3, plus at Brezal, Hondo de Abajo, Hornos, Soria 3.

\section{Psectrocladius sp.}

Larvae belonging to this genus were found at Carrizal 1, Siberio and Soria 3.

Rheocricotopus (Psilocricotopus) atripes (Kieffer, 1913) WP

Besides at the four localities given in Table 3, larvae of this species were found also at Brezal.

Thienemanniella sp. nr clavicornis (Kieffer, 1911) WP

Besides at the seven localities given in Table 3, larvae of this species were found also at Guayadeque, Hondo de Abajo, and Homos.

Chironominae

Chironomini

\section{Chironomus sp.}

Our light traps collected at least four unidentified species belonging to this genus. Besides at the five localities given in Table 3, larvae of this genus were found also at Carrizal 1, Coruña, Hornos, and Soria 3.
Dicrotendipes septemmaculatus (Becker, 1908) Af \& WP

Adults of this species were collected in the light traps at Azuaje and Tirajana in November 1995. Larvae of this genus were found at Carrizal 1 and Siberio.

Phaenopsectra sp.

Besides at the three localities given in Table 3, larvae of this genus were found also at Brezal.

Polypedilum (Pentapedilum) sp. nr sordens (van der Wulp, 1874) WP

The adult material included probably at least five different species of this genus, of which only one could be associated with a species name. Larvae of the genus were found only at Moya.

Stictochironomus sp. nr maculipennis (Meigen, 1818) WP

Adults of this species were collected in the light traps at Tirajana in both 1994 and 1995. Larvae of this genus were found only at Tejeda.

Tanytarsini

Cladotanytarsus ?atridorsum Kieffer, 1924 WP

Adults probably belonging to this species were collected in the light trap at Azuaje in 1995. Larvae of this genus occurred at Mina 3, Moya and Soria 3.

Micropsectra atrofasciata (Kieffer, 1911) Af \& WP

Adults of this species were taken in the light traps at Mina 2 and Soria 1. Larvae belonging to this genus were very abundant at Mina 3, and found also at Coruña, Hondo de Abajo, Hornos, and Peréz dam. A few pupae from Mina 3 were identified as this species.

Micropsectra ?notescens (Walker, 1856) WP

A pupa from Hornos probably represents this species.

Rheotanytarsus sp.

Larvae of this genus occurred at Azuaje, Carrizal 1-2, Cernicalos, Guayadeque, Siberio, and Tejeda (Tables 3-4).

Tanytarsus chinyensis Goetghebuer, 1934 WP

Adults of this species were collected in the light traps at Azuaje and Tirajana in 1995. Larvae belonging to this genus were found only at Moya (Table 3).

Virgatanytarsus albisutus (Santos Abreu, 1918) Af

Adults of this species were collected in the light traps at Azuaje and Tirajana in November 1995. Larvae belonging to this genus were found at Azuaje and Carrizal 1-2.

Stratiomyidae

Oxycera grancanariensis Frey, 1936 Ca (LP T)

This species was first described from a stream near Las Lagunetas at $1000 \mathrm{~m}$ asl (Frey 1936). More recently, (Rozkosny \& Báez 1983) gave a record from Azuaje 1973. We collected larvae of this species at Azuaje, Cernicalos, Guayadeque, and Mina 3.

Empididae

Clinocera amarantha Becker, $1908 \mathrm{Ca}$ (LP T)

Frey (1936) recorded this species from Las Lagunetas and Moya. We know of no subsequent records from Gran Canaria. 


\section{Hemerodromia claripennis Frey, 1958 Ca (LP T)}

Larvae or pupae of this species were found at Coruña, Mina 3 , and Soria 2. It was seemingly not known from Gran Canaria earlier.

\section{Dolichopodidae}

Unidentified larvae belonging to this family were found at Hondo de Abajo and Soria 2.

Ephydridae

Adults of the following two species were present in our light traps : Ephydra macellaria Egger, 1862, at Azuaje in 1995, and Scatella stagnalis (Fallén, 1813) at Tirajana in November 1995 .

\section{Muscidae}

The aquatic forms of this family belong to the tribe Limnophorini, of which the Canarian species were revised by Báez (1981). We here list only the species with supposedly aquatic larvae. Our own material of larvae and pupae has not been identified to species.

Limnophora quatema (Loew, 1852) Af

This species was recorded from Gran Canaria by Becker (1908).

\section{Limnophora obsignata (Rondani, 1866) Me}

This species was recorded from Gran Canaria by Frey according to Báez (1981).

Limnophora setinerva Schnabl \& Dziedzicki, $1911 \mathrm{Me}$

Frey (1936) recorded this species from Las Lagunetas.

Limnophora beckeri (Stein, 1908) Pal

This species was recorded from Gran Canaria by Frey according to Báez (1981).

Limnophora bipunctata (Stein, 1908) Me

This species was collected at Azuaje in 1973 (Báez 1981).

Limnophora nitidithorax (Stein, 1908) C (LP T)

This species was collected at Azuaje in 1973 (Báez 1981).

\section{Local diversity and similarity}

In total, our benthic samples from Gran Canaria included 123 taxa of limnic insects and gastropods. Of these, 98 taxa were represented in the semiquantitative net samples, including almost 9,000 identified specimens. Looking at these samples only, local species richness ranged from 11 to 36 species, with Tejeda 1994, Moya and Mina 3 being the richest localities (Table 5). As these values are based on pooled data from riffle and pool samples, that from Siberio is underestimated due to the absence of riffle samples from this locality. When the species found only in non-standardized collections at the same sites are added, Tejeda 1994 stands out with 48 , followed by Siberio with 47, Moya with 46, and Azuaje 1994 with 44 species (Table 5).
Table 5. Summations of individuals and species from standardized net samples taken in Gran Canarian streams. Species collected in qualitative samples from the same localities were included in one of the species counts. Diversity is given as Fisher's $\alpha$.

Tableau 5. Totaux des individus et espèces à partir de récoltes au filet standard dans les cours d'eau de Gran Canaria. Les espèces récoltées dans des prélèvements qualitatifs sur les mêmes sites sont comptabilisées dans un total des espèces. La diversité spécifique est donnée selon $\alpha$ de Fisher.

\begin{tabular}{lrrrrr}
\hline & \multicolumn{5}{c}{ No. of species } \\
\cline { 3 - 5 } Locality & No. of inds & Qual. excl & Qual. incl & Fisher's ax \\
\hline & & & & \\
Azuaje94 & 781 & 28 & 44 & 5.678 \\
Azuaje95 & 544 & 25 & 27 & 5.411 \\
Cernicalos94 & 1025 & 24 & 32 & 4.399 \\
Cernicalos95 & 554 & 27 & 26 & 5.939 \\
Coruña94 & 1369 & 28 & 35 & 4.983 \\
Mina 2 & 350 & 15 & 18 & 3.186 \\
Mina 3 & 1306 & 36 & 40 & 6.850 \\
Moya94 & 404 & 36 & 46 & 9.555 \\
Siberio & 197 & 19 & 47 & 5.187 \\
Soria 1 & 77 & 11 & 12 & 3.512 \\
Soria 2 & 521 & 22 & 35 & 4.654 \\
Tejeda94 & 297 & 35 & 48 & 10.310 \\
Tejeda95 & 892 & 28 & 28 & 5.495 \\
Tirajana94 & 617 & 30 & 35 & 6.595 \\
\hline
\end{tabular}

Using Fisher's $\alpha$ (Magurran 1988) calculated from the semiquantitative net samples as a diversity estimate, Tejeda 1994 and Moya stand out as the two most diverse localities (Table 5).

It is more difficult to evaluate the different number of species collected at the visits when only non-standardized collecting methods were used (Table 4), as the results are highly dependent on the investigation effort. However, the two richest sites were apparently Soria 3 in 1994 and Hondo de Abajo with 37 and 31 taxa respectively.

Looking at the distribution of the individual taxa among the studied streams, no nested pattern was found (Patterson \& Atmar 1986; RANDOM 1 test). Such a pattern would not be expected as the richest stream harboured only $39 \%$ of the total number of taxa found.

Faunal similarities among the localities where semiquantitative net samples were taken were estimated by UPGMA clustering. When based only on these quantitative data, some of the sites clustered at relatively high similarities (Fig. 2A) with Cernicalos 1995, Mina 2, Moya, Siberio, Soria 1-2, and Tirajana making up one cluster. Most disparate in this analysis were Coruña, Cernicalos 1994, and Mina 3. 
Similarities between localities were much lower when the analysis was based on presence/absence data and also the results of the non-standardized sampling was included (Fig. 2B). Here Soria 2 clustered with Tirajana, and the two Cernicalos visits with Mina 2, whereas Moya and Mina 3 were most disparate.

\section{Discussion}

Four of the seven gastropod species found are introduced exotics. Ancylus striatus is endemic to Madeira and the Canaries, and is very common in Gran Canaria. Of the seven recorded mayfly species, only Baetis canariensis and $B$. pseudorhodani are Canarian endemics. Both of these species were found at a fair number of localities.

Generally, we found odonate larvae at few of our localities. However, they may have a more frequent occurrence in ponds and other habitats not sampled in our survey. Zygonyx torrida, however, is strictly rheophilic and today seemingly restricted to Azuaje. Only Sympetrum nigrifemur is endemic to the Atlantic Islands, and this species seems presently very rare in Gran Canaria, and was found by us only at Soria 3.

Our material includes specimens of nine of the 15 species of aquatic Heteroptera known from Gran Canaria. Mesovelia vittigera and Microvelia gracillima are here reported from this island for the first time. Of the Canarian endemics, Notonecta canariensis was quite common, whereas Velia lindbergi was found at relatively few sites, and abundantly only at Cernicalos.

The aquatic Coleoptera are rich in species in Gran Canarian streams; with only Diptera being more diverse. Of the 37 beetle species known from Gran Canaria, only Aulonogyrus striatus and Eretes sticticus are seemingly extinct from this island. Stictonectes canariensis and Limnebius canariensis are the only species considered as local endemics. However, the endemic status of the Stictonectes species has been doubted (Balke et al. 1990). The present status of Limnebius canariensis is very uncertain as the only stream in which we did find it was totally dry in 1995. Moreover, the only other stream in which it ever has been found, in Bco. Tirajana, had only isolated pools in 1995 . Of the nine Canarian endemics Hydroporus errans, Graptodytes delectus, Limnebius gracilipes, and Laccobius canariensis all are relatively common in Gran Canaria, despite the pronounced habitat fragmentation. The other five species have very restricted distributions in Gran Canaria today. Hydrotarsus pilosus, Ochthebius lapidicola and Hydraena serricollis all seem to prefer madicolous habitats, whereas Nebrioporus canariensis and Anacaena haemorrhoa are confined to streams.

The number of records of Trichoptera species from Gran Canaria is relatively low. We found ten of the 14 species known from the island. Orthotrichia angustella, Oxyethira falcata, Stactobia storai and the unidentified Polycentropus species are here all recorded from Gran Canaria for the first time. The presence of species pairs in four of the genera has made it difficult to estimate the current status of the species as most of our records are based on larvae, which could only be identified to the genus level. As we did not find any specimens of the local endemics Stactobia freyi, Tinodes arcuata or Oecetis canariensis their present status in Gran Canaria must be considered uncertain. Species richness was highest at Azuaje and Cernicalos with four species each. However, the rarer local endemics occurred at different sites, e.g. Agapetus adejensis at Cernicalos, Stactobia storai at Guayadeque, and Polycentropus sp. at Siberio and Tirajana.

Our list includes some 80 taxa of aquatic Diptera. This is an underestimate of their true diversity as some families like Ceratopogonidae and Ephydridae are not dealt with at the species-level. The 38 recorded chironomid taxa are hard to evaluate as so many of them could be identified only to the genus level. Our Gran Canarian record of Bryophaenocladius cuneiformis is noteable as this species was earlier known only from Tenerife.

Our coverage of the family Simuliidae is probably quite good. The Canarian endemic Simulium guimari is seemingly not very rare in Gran Canaria today. In contrast, the local endemic $S$. paraloutetense must be regarded as very close to total extinction. Our only record was from a very small trickle at Guayadeque.

Besides insects and gastropods, turbellarians and annelids were the most frequently encountered benthic macroinvertebrates. The turbellarians were represented in most streams by an unidentified species in the Dugesia gonocephala species group. Oligochaetes were not identified to species. Of the Hirudinea, we observed the same two species as in Tenerife (Malmqvist et al. 1995). A few specimens of Limnatis nilotica (Moquin-Tandon, 1826) were observed at Azuaje in 1994, whereas Helobdella stagnalis (Linnaeus, 1758) was collected at the following 4 localities : Carrizal 1 , Coruña, Siberio and Soria 3. Our records of Ostracoda have been published separately (Malmqvist et al. 1997).

With respect to the species richness of different taxa, the similarity with the stream fauna of Tenerife is striking (Malmqvist et al. 1993). The majority of species occur on both islands. Excluding Diptera, only six of the species recorded from Tenerife are seemingly ab- 

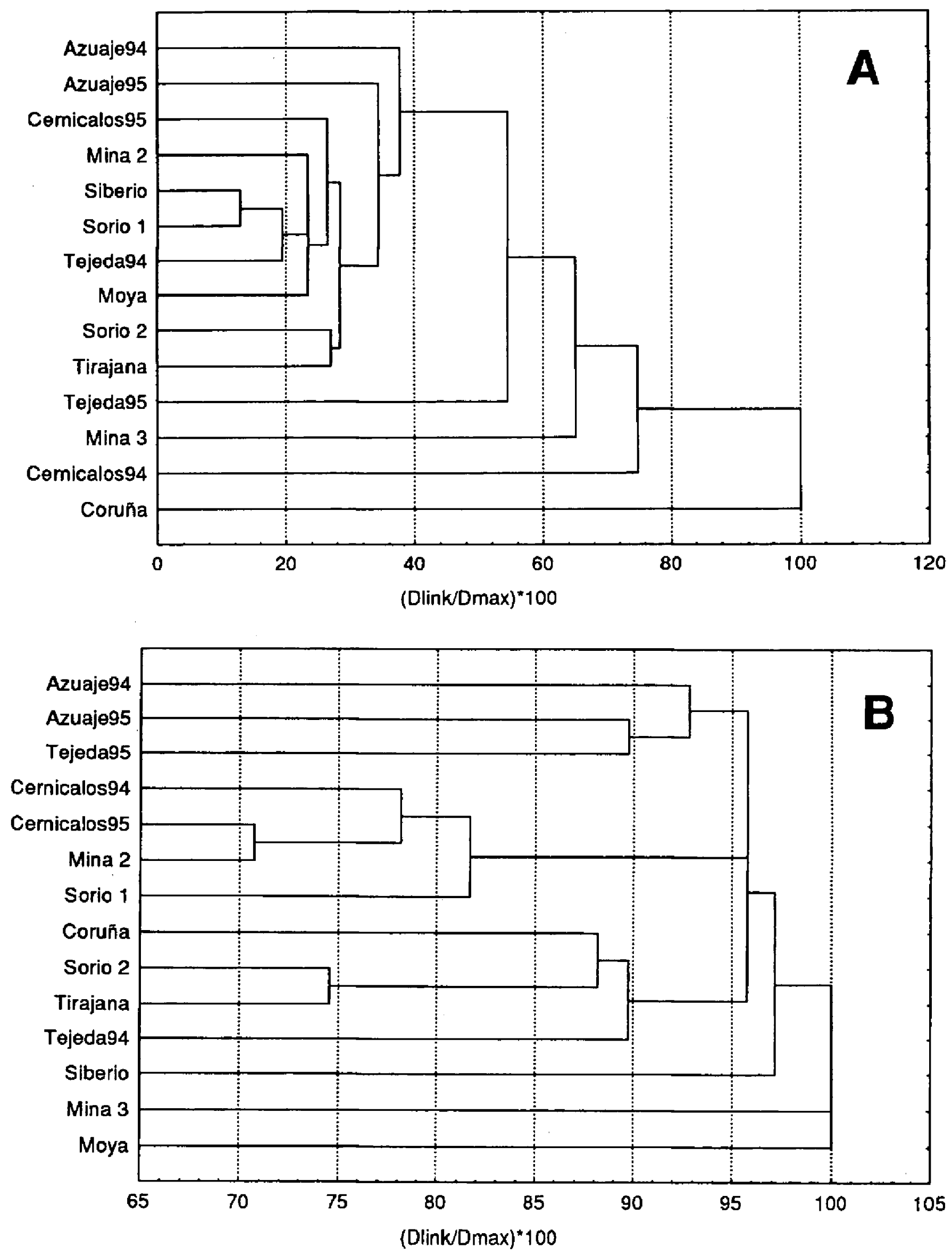

Fig. 2A-B. Similarity of stream localities in Gran Canaria based on their gastropod and insect faunas as derived from UPGMA clustering. -A. Based on quantitative data including 98 taxa. -B. Based on presence/absence data of 114 taxa. Visits from different years treated as separate cases.

Fig. 2A-B. Affinité coenotique des sites de Gran Canaria fondée sur leurs faunes d'insectes et de gastéropodes à partir d'une UPGMA cluster analyse -A. Basée sur les données quantitatives de 98 taxa -B. Basée sur les données présence/absence de 114 taxa. Les récoltes des différentes années sont traitées séparément. 
sent from Gran Canaria. On the other hand, 13 of the species known from Gran Canaria have not been found in Tenerife. As in Tenerife, streams in Gran Canaria share relatively few species making each site unique with respect to community structure. The only species seemingly endemic to Gran Canaria are : Stictonectes canariensis, Limnebius canariensis, Stactobia freyi, Tinodes arcuatus and Simulium paraloutetense. With the exception of $S$. canariensis all of them are already extinct or perhaps very close to extinction with small populations found at isolated localities.

Azuaje, Cernicalos, and La Mina may be the only three permanent streams now remaining in Gran Canaria. Water may also be present permanently at other localities, either as pools or small springs. Permanent pools may exist locally due to deep basins in rock, or where hypogean stream water reaches the surface.

We have earlier stressed the vulnerability of the freshwater fauna in Tenerife (Malmqvist et al. 1995). The present study shows that the situation in Gran Canaria is no better and we think setting up and implementing a conservation plan for freshwater macroinvertebrates would be an urgent task.

\section{Acknowledgements}

Dr. Peter Langton provided taxonomic help with the Chironomidae, and Dr. Dom Chaloner helped us with the processing of them. Dr. Ted von Proschwitz, Gothenburg, kindly identified all the gastropods. Dr. Frank Johansson, Umeă, kindly identified our odonate larvae. Dr. Rüdiger Wagner, Schlitz, provided help in the identification of Empididae and Psychodidae. Dr. Hans Malicky, Lunz am See, identified all our adult caddis flies. Dr. Göran Englund, Umeå, kindly operated a light trap for us one evening. Dr. S. Kholin, Vladivostok, provided computer software for calculating Fisher's $\alpha$ and analysing nestedness. Dr. A. Thomas, Toulouse, kindly prepared the French abstract for us.

\section{References}

Alarie Y. \& Nilsson A. N. 1997.-Larvae of Stictonectes Brinck: Generic characteristics, description of $S$. canariensis Machado, and analysis of phylogenetic relationships with other genera of the tribe Hydroporini (Coleoptera : Dytiscidae). Coleopts. Bull., $51: 120-139$.

Armitage P. D., Blackbum J. H., Nilsson A. N. \& Malmqvist B. 1995.- Chironomidae in freshwater habitats in Tenerife, Canary Islands. In : P. Cranston (ed.) : Chironomids -from genes to ecosystems. CSIRO Publ., Melbourne : 379-388.

Baena M. \& Báez M. 1990.— Los Heterópteros acuáticos de las Islas Canarias (Heteróptera; Nepomorpha, Gerromorpha). Vierea, $19: 233-244$.

Báez M. 1981.— Dipteros de Canarias VIII : Tribus Limnophorini y Coenosiini (Dipt., Muscidae). Anuario Estud. Atlant., 27 : 619 670

Báez M. 1985.- Las libelulas de las Islas Canarias. Enciclopedia Canaria, Aula de Cultura de Tenerife, Litografia A. Romero, Santa Cruz de Tenerife : 48p.

Báez M. \& Fernandez J. M. 1980.- Notes on the mosquito fauna of the Canary Islands. Mosq. Syst., $12: 349-355$.
Balke M., Hendrich L. \& Cuppen J. G. M. 1990.- Wasserkäfer von den Islas Canarias (Coleoptera : Haliplidae, Dytiscidae, Gyrinidae, Hydrochidae, Hydrophilidae, Hydraenidae, Dryopidae). Entomofauna, $11: 349-373$.

Becker T. 1908.- Dipteren der Kanarischen Inseln. Mitt. zool. Mus. Berlin, 4 : 1-180.

Belle J. 1982.- Odonata collected in the Canary Islands. Ent. Ber., $42: 75-77$.

Brauer F. 1901 (1900).-- Über die von Prof. O. Simony auf den Canaren gefundenen Neuroptera und Pseudoneuroptera (Odonata, Corrodentia et Ephemeridae). Sitzb. math.-nat. kais. Akd. Wiss., 109 (1) : 464-477.

Crosskey R. W. 1988. - Taxonomy and geography of the blackflies of the Canary Islands (Diptera : Simuliidae). J. nat. Hist., 22 : 321-355.

Frey R. 1936.-Die Dipterenfauna der Kanarischen Inseln und ihre Probleme. Comm. biol. Soc. sci. fenn., 6 (1) : 1-237.

Gardner A. E. 1960.— Odonata, Saltatoria and Dictyoptera collected by Mr. E.S.A. Baynes in the Canary Islands 1957 to 1959 . Entomologist, 93 : 128-131.

Hemmingsen A. M., Mannheims B. \& Nielsen P. 1959.— Notes on craneflies (Tipulidae) collected by Dr. Axel M. Hemmingsen in the Canary Islands. Ent. Meddr, $29: 71-77$.

Jäch M. A. 1993. - Taxonomic revision of the Palearctic species of the genus Limnebius Leach, 1815 (Coleoptera : Hydraenidae). Koleopt. Rundsch., 63 : 99-187.

Jong H. de 1987. - Keys for the identification of the Tipuloidea (Insecta, Diptera) recorded for the Canary Islands. Eos, 63 : 73-92.

Lackschewitz P. 1936.-Fam. Tipulidae, Fam. Limoniidae, p. 5-8. In : R. Frey (ed.), Die Dipterenfauna der Kanarischen Inseln und ihre Probleme. Comm. biol. Soc. sci. fenn. 6(1) : 1-237.

Lindberg H. 1953.— Entomologische Ergebnisse der finnländischen Kanaren-Expedition 1947-1951. No. 1. Hemiptera Insularum Canariensium. Systematik, Ökologie und Verbreitung der Kanarischen Heteropteren und Cicadinen. Comm. biol. Soc. sci. fenn., 14 (1) : 1-304.

Machado A. 1987a.-Bibliografía entomológica Canaria. Instituto de Estudios Canarios, La Laguna : 295p.

Machado A. 1987b. - Los Ditiscidos de las Islas Canarias (Coleoptera, Dytiscidae). Instituto de Estudios Canarios, La Laguna : 81 p.

Malmqvist B., Meisch C. \& Nilsson A. N. 1997.- Distribution patterns of freshwater Ostracoda (Crustacea) in the Canary Islands with regards to habitat use and biogeography. Hydrobiol., 347 : 159-170.

Malmqvist B., Nilsson A. N. \& Báez M. 1995.- Tenerife's freshwater macroinvertebrates : status and threats (Canary Islands, Spain). Aquatic Cons., 5 : 1-24.

Malmqvist B., Nilsson A. N., Báez M., Armitage P. D. \& Blackbum J. 1993.- Stream macroinvertebrate communities in the island of Tenerife. Arch. Hydrobiol., 128 : 209-235.

Magurran A. E. 1988.-Ecological diversity and its measurement. Princeton, New Jersey : $179 \mathrm{p}$.

Marzol M. V. 1984.-El clima. - In : Geografía de Canarias, tomo 1. Ed. Interinsular Canaria. Tenerife : 158-202.

McLachlan R. 1883.- The Neuroptera of Madeira and the Canary Islands. J. Linn. Soc. Lond. Zool., $16:$ 149-183.

Müller-Liebenau I. 1971.-- Ephemeroptera (Insecta) von den Kanarischen Inseln. Gewässer und Abwässer, 50/51 : 7-40.

Navas R. P. L. 1906. - Catálogo descriptivo de los Insectos Neurópteros de las Islas Canarias. Rev. Acad. Cienc. exactas fis. nat., 4 : $687-706+1 \mathrm{pl}$.

Nybom O. 1948.- The Trichoptera of the Atlantic Islands. Comm. biol. Soc. sci. fenn., 8 (14) : 1-19. 
Nybom O. 1954. - Entomological results of the Finnish expedition to the Canary Islands, 1947-1951. No. 9. Some additions to the trichopterous fauna of the Canary Islands. Comm. biol. Soc. sci. fenn., 14(9) : 1-3.

Orchymont A. d'. 1940.- Les Palpicornia des Îles Atlantiques. Mém. Mus. r. Hist. nat. Belg., (2) $20: 1-87$.

Patterson B. D. \& Atmar W. 1986. - Nested subsets and the structure of insular mammalian faunas and archipelagos. Biol. J. Linn. Soc., $28: 65-82$.

Rosenzweig M. L. 1995.- Species diversity in space and time. Cambridge University Press, Cambridge : $436 \mathrm{p}$.

Storå R. 1936.- Fam. Dixidae, Culicidae, Chironomidae, Thaumaleidae, Ceratopogonidae, Simuliidae, p. 19-39. In : R. Frey (ed.) : Die Dipterenfauna der Kanarischen Inseln und ihre Probleme. Comm. biol. Soc. sci. fenn., 6 (1) : 1-237.

Tamanini L. 1954.- Resultati entomologici della specizione finlandese all Canarie. No. 5. Velia lindbergi $\mathrm{n}$. sp. e V. maderensis Noualhier (Hem., Het., Veliidae). Comm. biol. Soc. sci. fenn., 14 (5) : $1-7$
Theowald B. 1977.- Die Tipuliden von Makaronesien (Insecta, Diptera, Tipulidae). Ein systematischer und zoogeographischer Beitrag zur Kenntnis von Inselfaunen. Beaufortia, 26 (335) : 153204.

Tonnoir M. A. 1921 - Faune entomologique des Iles Canaries. Séjour de M.P. Lesne dans la Grande Canarie (1902-1903). III. Diptères : Psychodidae. Bull. Mus. natn. Hist. nat., Paris, 27 : 296297.

Valle K. J. 1935.- Die Odonaten der Kanarischen Inseln. Comm. biol. Soc. sci. fenn., 6 (5) : 1-7.

Valle K. J. 1955. - Zygopteren (Odonata) von den Kanarischen Inseln. Annls ent. fenn., $21: 182$.

Wollaston T. V. 1864. - Catalogue of the Coleopterous insects of the Canaries in the collection of the British Museum. Taylor \& Francis, London : 648p.

Zimmermann G. 1984. - Zur Wasserwanzenfauna der Kanarischen Inseln und deren zoogeographische Beziehungen zum Festland und anderen Inselngruppen (Insecta : Heteroptera : Hydrocorisae). Cour. Forsch. Inst. Senckenberg, $71: \$ 3-60$. 